"Novel non-viral gene delivery systems composed of dendron functionalized nanoparticles prepared from nano-emulsions as non-viral carriers for antisense oligonucleotides" Fornaguera, C., Grijalvo, S., Galán, M., Fuentes-Paniagua, E., de la Mata, F.J., Gómez, R., Eritja, R., Calderó, G., Solans, C. Int. J. Pharm., 478(1), 113-123 (2015). doi: 10.1016/j.ijpharm.2014.11.031

\title{
Novel non-viral gene delivery systems composed of carbosilane dendron functionalized nanoparticles prepared from nano-emulsions as non-viral carriers for antisense oligonucleotides
}

\author{
Cristina Fornaguera ${ }^{a, b}$, Santiago Grijalvo ${ }^{a, b}$, Marta Galán ${ }^{b, c}$, Elena Fuentes-Paniagua a,c, \\ Francisco Javier de la Mata ${ }^{b, c}$, Rafael Gómez ${ }^{b, c}$, Ramon Eritja ${ }^{a, b}$, Gabriela Calderó ${ }^{a, b}$, \\ Conxita Solans ${ }^{\mathrm{a}, \mathrm{b}}$,
}

anstitute of Advanced Chemistry of Catalonia (IQAC-CSIC), C/Jordi Girona, 18-26, 08034, Barcelona, Spain;

${ }^{\mathrm{b}}$ CIBER-BBN Networking Centre on Bioengineering, Biomaterials and Nanomedicine (CIBERBBN), Spain;

'Group of Dendrimers for Biomedical Applications, University of Alcalá (GDAB-UAH), Alcalá de Henares, Madrid, Spain.

ABSTRACT. The development of novel and efficient delivery systems is often the limiting step in fields such as antisense therapies. In this context, poly(D,L-lactide-co-glycolide) acid (PLGA) nanoparticles have been obtained by a versatile and simple technology based on nanoemulsion templating and low-energy emulsification methods, performed in mild conditions, providing good size control. O/W polymeric nano-emulsions were prepared by the phase inversion composition method at $25 \stackrel{\circ}{\circ} \mathrm{C}$ using the aqueous solution/polysorbate $80 /[4 \mathrm{wt} \%$ PLGA in ethyl acetate] system. Nano-emulsions formed at oil-to-surfactant $(\mathrm{O} / \mathrm{S})$ ratios between 10/90-90/10 and aqueous contents above $70 \mathrm{wt} \%$. Nano-emulsion with $90 \mathrm{wt} \%$ of aqueous solution and $\mathrm{O} / \mathrm{S}$ ratio of 70/30 was chosen for further studies, since they showed the appropriate characteristics to be used as nanoparticle template: hydrodynamic radii lower than $50 \mathrm{~nm}$ and enough kinetic stability. Nanoparticles, prepared from nano-emulsions by solvent evaporation, showed spherical shape, sizes about $40 \mathrm{~nm}$, negative surface charges and high stability. The as-prepared nanoparticles were functionalized with carbosilane cationic dendrons through a carbodiimide-mediated reaction achieving positively charged surfaces. Antisense oligonucleotides were electrostatically attached to nanoparticles surface to perform gene-silencing studies. These complexes were non-haemolytic and non-cytotoxic at the concentrations required. The ability of the complexes to impart cellular uptake was also 
promising. Therefore, these novel nanoparticulate complexes might be considered as potential non-viral carriers in antisense therapy.

Keywords. Nano-emulsion; PIC emulsification method; PLGA nanoparticles; Cationic Dendron; Non-viral gene delivery systems; Antisense therapy.

\section{Introduction}

Nowadays, gene therapy has an increasing interest in the biomedical field due to the ability of DNA to modulate the expression of human genes, thus enabling, for example, the down- regulation of genes involved in diseases. In this context, since their discovery, both antisense and RNA interference oligonucleotide therapies have been considered as potential therapeutics due to the ability to selectively modulate the expression of an individual gene (Zamecnik and Stephenson, 1987; Fire et al., 1998; Loke et al., 1989). However, the delivery of nucleic acids represents a challenge related to their low stability against nuclease activity and their low intracellular penetration (Fattal et al., 1998; Chavany et al., 1992). To circumvent these problems, viral and non-viral vectors have been used. Viral vectors have often high transfection efficiencies, although there are several concerns about their use in human therapy such as an increased immune response, the possible recombination of oncogenes and the difficulty in scale-up (Giacca and Zacchigna, 2012). For these reasons, non-viral vectors have emerged as promising carriers due to their reduced immune response, low toxicity and their safety in comparison to viral vectors (Puras et al., 2014; Meyer et al., 1998; Newland et al., 2012; Li et al., 2011).

Among multiple examples of non viral vectors, polymeric nanoparticles are advantageous systems because they have a nanometric size and their surface can be efficiently modified to achieve the required properties (Newland et al., 2012; Torchilin, 2006). Examples of polymeric nanoparticles containing oligonucleotides have been previously reported. However, their preparation often requires the use of complex methodologies (e.g. high energy input, extreme $\mathrm{pH}$ values, high temperatures, tedious purification steps, etc.) (Patil and Panyam, 2009). Nano-emulsion templating represents a versatile and simple technology for the fabrication of nanoparticles for different biomedical uses (Calderó et al., 2011; Landfester, 2001; Calderó and Solans, 2012) by which the above mentioned disadvantages can be avoided. Nano-emulsions are emulsions with droplet sizes typically between 20 and $200 \mathrm{~nm}$ (Solans et al., 2005; Solans and Solè, 2012) that can be controlled by appropriate selection of system components, composition and process parameters. Due to their nanometric size, they show a transparent to translucent appearance and are stable against creaming and/or sedimentation. Although they are thermodynamically unstable systems and therefore, an energy input is required for their formation, the source of the required energy can be internal, rising from the intrinsic chemical energy of the system components which is released during the emulsification process. These are the so-called low-energy emulsification methods (Solans and Solè, 2012) which awake increasing attention because they are advantageous (good size control, mild process conditions) compared to the conventional high-energy methods. 
a)

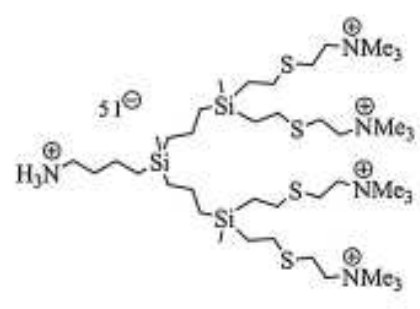

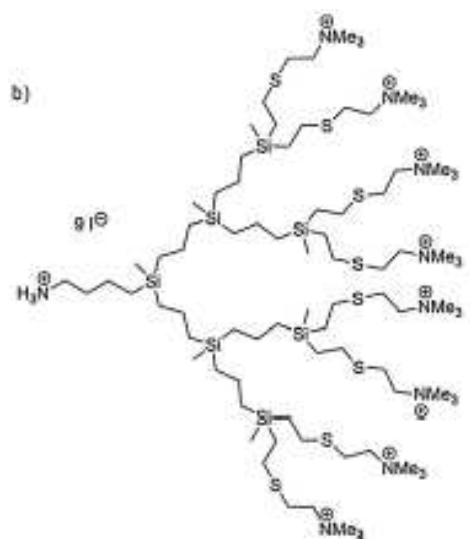

Figure 1. Chemical structure of carbosilane dendrons of: (a) second and (b) third generation

One of the most appropriate low-energy emulsification method for biomedical applications is the so-called phase inversion composition (PIC) method (Solans and Solè, 2012), The emulsifi-cation is triggered by phase transitions produced changing the composition of the system at constant temperature whereby phase inversion takes place. This methodology, besides allowing size control, can be carried out at the desired temperature; it requires simple equipment and it is easily scalable (Sadurní et al., 2005).

Polymeric nanoparticles can be prepared from nano-emulsion templating by the use of a preformed polymer (dissolved in the dispersed phase) followed by solvent evaporation. The strategy using a preformed polymer is usually simple, fast (by choosing appropriate system components the as-prepared nanoparticle dispersion can be used, avoiding tedious purification steps) and reproducible (Vauthier and Bouchemal, 2009). In this work, poly(D, Llactide-co-glycolide) acid (PLGA) has been used because it is biocompatible, biodegradable and FDA approved (Dinarvand et al., 2011).

Before decorating our PLGA nanoparticles with oligonucleo- tides, additional positive charges should be added into the nanoparticle surface. This strategy would allow formation of complexes that might protect oligonucleotides from nuclease attack and hence improve their cellular uptake properties (Fattal et al., 1998; Chavany et al., 1992). For example, the introduction of cationic polymers and chitosans are often the general strategies. However, several concerns regarding cytotoxic effects induced either by the presence of amine groups which are susceptible to protonation at acidic $\mathrm{pH}$. The internalization of protonable amine groups through receptor-mediated endocytosis enables the fusion of these groups with endosomes, causing an endosomal rupture, which could mediate a gene delivery to the cytoplasm, sometimes favoring oligonucleotides delivery (Basarkar and Singh, 2007; Kumar et al., 2004). Nevertheless, accumulation problems due to their low biodegradation have been widely reported (Tahara et al., 2009). Consequently, the search of new biocompatible and nontoxic cationic entities to decorate nanoparticles is necessary. In this context, cationic carbosilane dendrons (Fig. 1) may constitute an advantageous approach (Fuentes-Paniagua et al., 2013), as it has been reported that they show good biocompatibility with cells (Ortega et al., 2006). 
In this work, we report the use of as-prepared polymeric nanoparticle dispersions obtained from nano-emulsion templating using a low-energy emulsification method without further washing steps and their multi-functionalization by using cationic dendrons to generate complexes with phosphorothioate oligonucleotides in order to study their silencing activities and the ability of these systems to mediate cellular uptake. This strategy would represent a novel approach to obtain new non-viral carriers for oligonucleotides.

Aqueous solution (W)

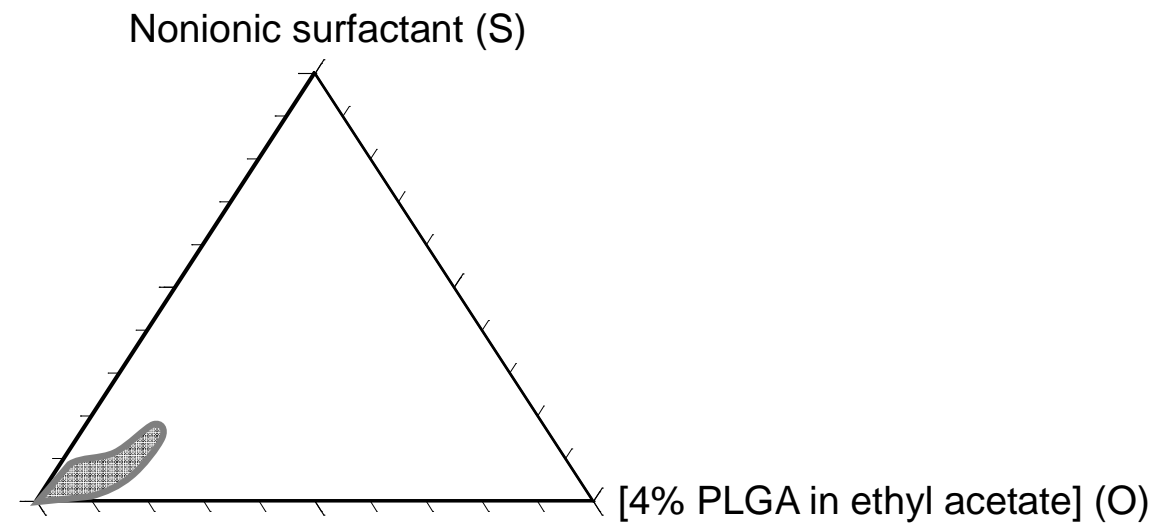

Fig. 2. O/W nano-emulsion region (grey area) of formation in the water/Polysorbate $80 /[4 \mathrm{wt} \%$ PLGA in ethyl acetate] system at $25 \stackrel{\circ}{\circ}$.

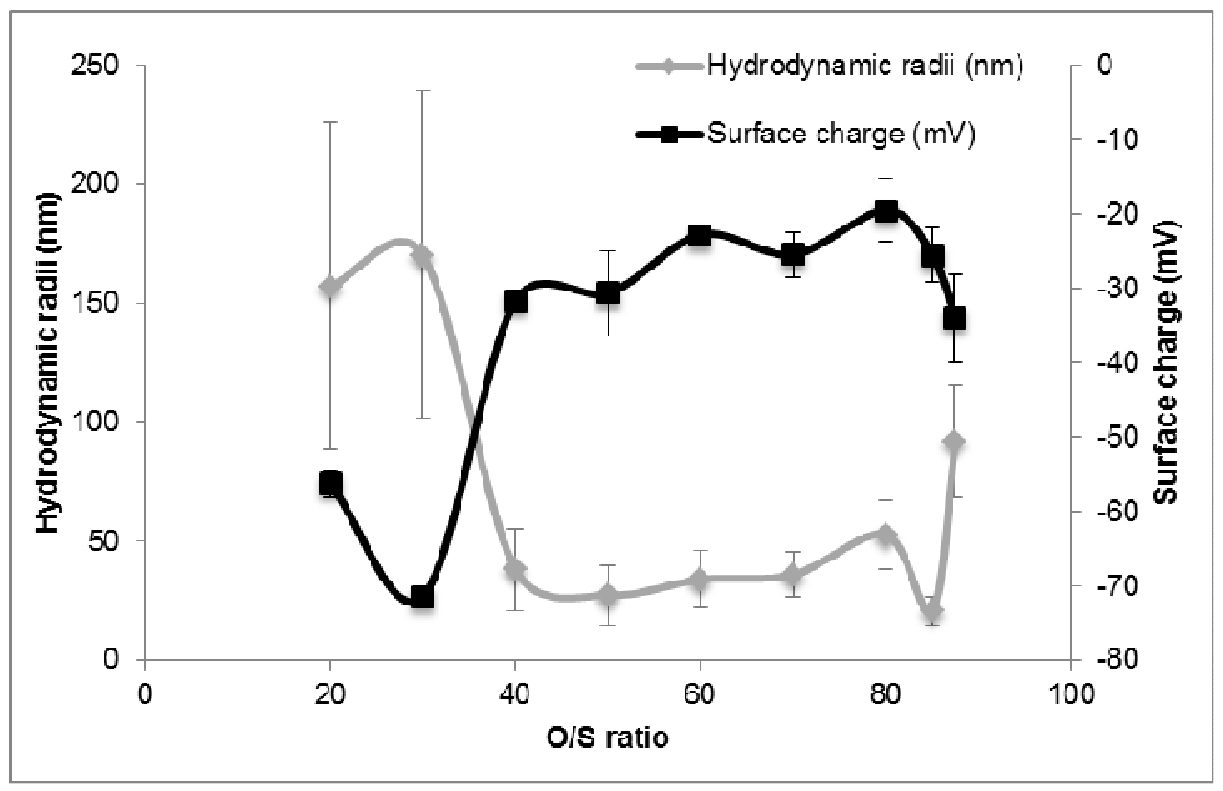

Fig. 3. Nano-emulsion hydrodynamic radii mean size and surface mean charge as a function of the $\mathrm{O} / \mathrm{S}$ ratio, at $90 \mathrm{wt} \%$ of water content. The vertical lines of each point indicate the standard deviation. 


\section{Experimental}

\subsection{Materials}

Pharmaceutical grade poly(D,L-lactide-co-glycolide) acid, Resomer $752 \mathrm{H}$, in the following abbreviated as PLGA (polystyrene equivalent molecular weight PSE-MW 10,000 $\mathrm{g} / \mathrm{mol}$, as determined by gel permeation chromatography) was purchased from Boehringer Ingelheim. The lactic to glycolic acid ratio was 75/ 25 and the end groups were free carboxylic acids. Ethyl acetate (Ph Eur grade) used as the organic volatile solvent was purchased from Merck. This generally recognized as safe (GRAS) solvent was chosen due to its low toxicity and suitable properties for evaporation (boiling point $=77^{\circ}$ C) (Rowe et al., 2006). The nonionic surfactant Polysorbate 80, an ethoxylated sorbitan monooleate with an HLB value of 15 (Rowe et al., 2006) was kindly provided by Croda. Sodium chloride ( $\mathrm{NaCl})$, sodium dihydrogenphosphate hydrate $\left(\mathrm{NaH}_{2} \mathrm{PO}_{4} \cdot \mathrm{H}_{2} \mathrm{O}\right)$, disodium mono-hydrogenphosphatedihydrate $\left(\mathrm{Na}_{2} \mathrm{HPO} \cdot 2 \mathrm{H}_{2} \mathrm{O}\right)$, sodium hydroxide $(\mathrm{NaOH})$, ortophosphoric acid $\left(\mathrm{H}_{3} \mathrm{PO}_{4}\right)$ which were used to prepare the aqueous electrolyte solution (phosphate buffered saline: PBS), as well as 1-ethyl3-(3-dimethylaminopropyl) carbodiimide $(E D C, M W=191.7 \mathrm{Da})$ and N-hydroxysuccinimide (NHS, MW = 115.09) were purchased from Merck. Poly(ethylene glycol) (PEG) (MW = $400 \mathrm{Da})$ and HEPES salt were purchased from Sigma-Aldrich. Water was Milli-Q filtered (resistivity of 18.2 $\mathrm{M} \Omega \mathrm{cm}$; surface tension $=72.8 \mathrm{nN} / \mathrm{m}$ ). Dual-luciferase reporter assay system was purchased from Promega. Cationic dendrons with a primary amine focal point, a carbosilane structure and a quaternary amine with a positive charge at each ramification were selected. The second and third generation dendrons were used (Fig. 1a and b, respectively). An antisense phosphorothioate oligonucleotide of 18 nucleotides (sequence 5'-CGT TTC CTT TGT TCT GGA-3'), directed against a fragment between 20 and 40 nucleotides of the mRNA of the Renilla luciferase gene, as well as a 18-mer scrambled antisense oligonucleotide sequence (sequence 5'-CTG TCT GAC GTT CTT TGT- 3') were purchased from Proligo (Sigma-Aldrich). Carbosilane cationic dendrons and antisense oligonucleotides (ASO) were prepared as previously reported (Tahara et al., 2009; Ortega et al., 2006; Grijalvo and Eritja, 2012).

\subsection{Methods}

\subsubsection{Nano-emulsion preparation}

Nano-emulsions were prepared by the phase inversion composition (PIC) method (Patil and Panyam, 2009; Calderó and Solans, 2012). $4 \mathrm{~g}$ of nano-emulsions were formed by stepwise addition of $0.16 \mathrm{M}$ electrolyte solution, (a phosphate buffered saline, PBS solution, which components are indicated in Section 2.1) to mixtures of surfactant and oil at $25 \stackrel{\circ}{\circ}$. The oil phase consists of $4 \mathrm{wt} \%$ of PLGA in ethyl acetate. The region of formation of O/W nanoemulsions was visually assessed, observing the samples under a spotlight. Those samples with transparent, translucent or slightly opaque aspect, having a bluish or reddish shine were considered as nano-emulsions. To distinguish them from micro-emulsions, samples were also prepared by mixing all components at once. The nano-emulsion formation region (Fig. 2) was drawn using the Microsoft Power Point software.

\subsubsection{Nanoparticle preparation from nano-emulsion templating}


Nanoparticle dispersions were prepared from nano-emulsions by the solvent evaporation method under reduced pressure (Desgouilles et al., 2003), using a Büchi R-215V Rotavapor. The conditions for ethyl acetate evaporation from about $4 \mathrm{~g}$ of nano-emulsion at 25 ㅇ $\mathrm{C}$ temperature were: a vacuum of 43 mbar with a rotation speed of $150 \mathrm{rpm}$ during 45 $\mathrm{min}$, in order to ensure a residual solvent content below $5000 \mathrm{ppm}$.

a)

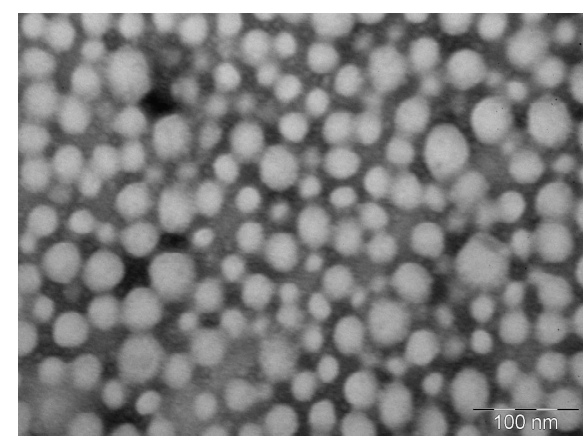

b) 30

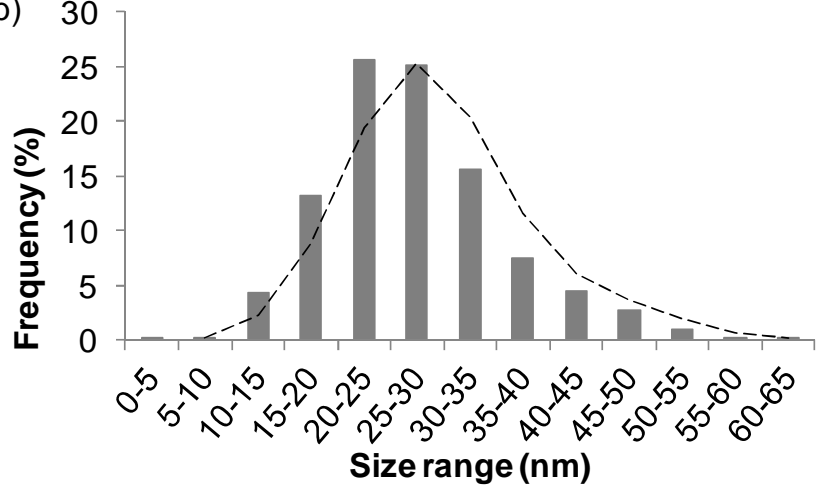

Fig. 4. TEM analysis of the nanoparticle dispersion obtained from the selected nano-emulsion. (a) Micrograph as the example of the nanoparticle shape and size and (b) diameter size distribution (diameter $=27.42 \pm 8.41 \mathrm{~nm}$ ).

\subsubsection{Droplet size characterization}

The mean droplet size and size distribution of nano-emulsions and the nanoparticle dispersions were determined by dynamic light scattering (DLS) with the 3D LS Spectrometer by LS Instruments (3D cross correlation multiple-scattering) equipped with a He-Ne laser (632.8 $\mathrm{nm}$ ) with variable intensity. Measurements were carried out at a scattering angle of 90, in triplicates, at $25 \stackrel{\circ}{ } \mathrm{C}$. Data were treated by cumulant analysis. The results are given as mean hydrodynamic sizes with the standard deviation and polydispersity indexes (PDI). At least 10 batches of the prepared nanoparticles were tested to assess the method reproducibility and the standard deviation. This characterization has been performed by the Nanostructured Liquid Characterization Unit of the Spanish National Research Council (CSIC) and the Biomedical Networking Center (CIBER-BBN), located at IQAC-CSIC.

\subsubsection{Nano-emulsion and nanoparticle stability}

Nano-emulsion and nanoparticle stabilities were assessed at 25 oC by visual examination of the samples as a function of time. Samples were considered stable if no macroscopic changes were observed. Stability was also assessed by the changes of size (determined by DLS) with time. 


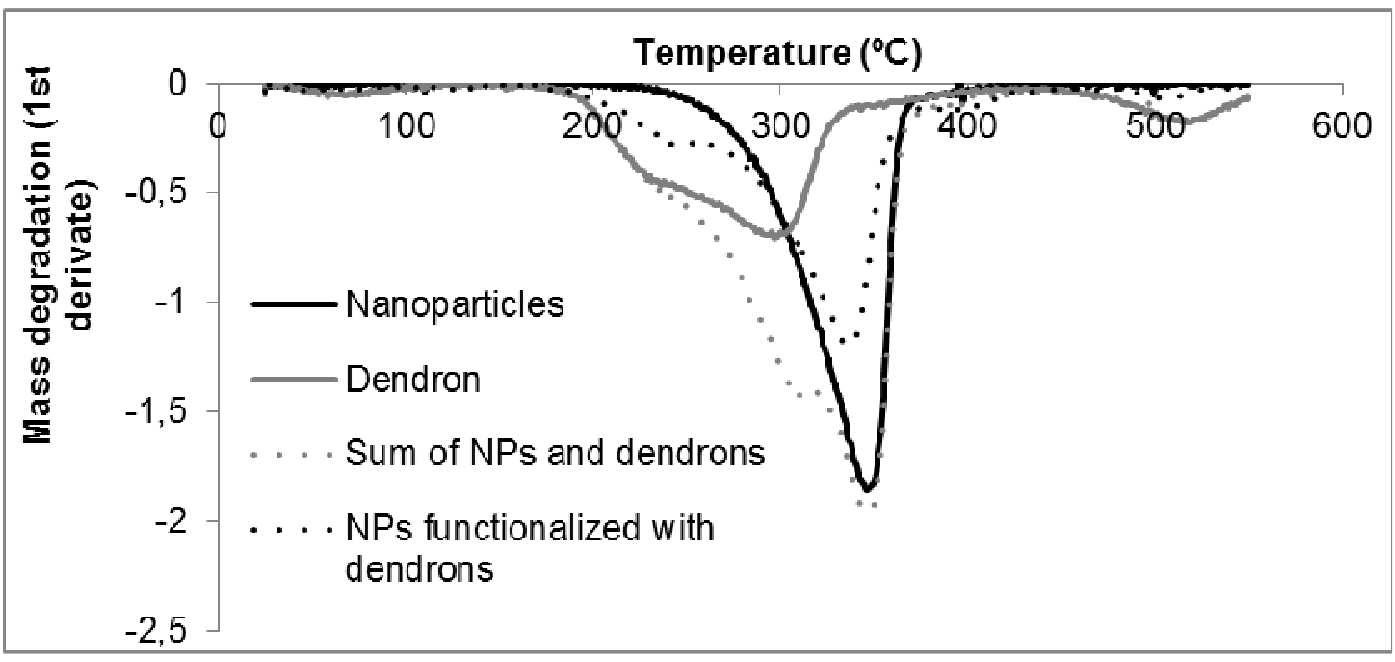

Fig. 5. Example of the results of the thermogravimetric assay performed with the second generation dendron. First derivative of the mass degradation of the samples.

\subsubsection{Surface charge determination}

The surface charge of the nanoparticles was assessed by electrophoretic mobility measurements with Zetasizer NanoZS instrument (Malvern Co., Ltd., UK), equipped with a HeNe red light laser $(\lambda=633 \mathrm{~nm})$.

\subsubsection{Transmission electron microscopy (TEM)}

Transmission electron microscopy (TEM) with a JEOL 1010 TEM (Jeol Korea Ltd.) was used to characterize the size and shape of the nanoparticle dispersions. Samples were prepared as described in the Supplementary Information (1S). The mean size and size distribution of the nanoparticles were determined by image analysis of pictures taken at different magnifications using the Image ${ }^{\circledR}$ software. Around 1000 nanoparticles selected from more than 40 TEM micrographs were measured for each sample. The results obtained with the software OriginPro81 are presented as mean and standard deviation.

\subsubsection{Covalent binding of dendrons to nanoparticles}

The functionalization of the nanoparticles with carbosilane dendrons was performed by using the carbodiimide reaction, linking the free carboxylic groups of the polymer along with the amine focal group of the dendrons through an amide bond. The carbodiimide reaction has been widely reported (Costantino et al., 2006; Betancourt et al., 2009), but in this work, some modifications have been introduced. Briefly, $4 \mathrm{~mL}$ of the as-prepared nanoparticle dispersion, dispersed in PBS, at a concentration of $3 \mathrm{mg} / \mathrm{g}(30 \mathrm{mM})$ of nanoparticles (NPs) were acidified $(\mathrm{pH}$ 5.5). The carboxylic groups of the PLGA were activated adding first NOethylcarbodiimide hydrochloride (EDC; 1 molar equivalent to PLGA, dissolved in PBS), vortexing, and then adding N-hydroxysuccinimide (NHS; 1 molar equivalent to PLGA, dissolved in PBS) to the nanoparticle dispersion during at least two hours at room temperature (25 으). After this time, activated nanoparticle dispersion was basified using sodium hydroxyde $1 \mathrm{M}$ 
$(\mathrm{pH} 8)$, as well as a solution of $1 \mathrm{~mL}$ of the dendron in milliQ water at the required nanoparticle/Dendron (N/D) molar ratio. Both components were mixed and stirred for at least $18 \mathrm{~h}$, at room temperature $(25 \stackrel{\circ}{\circ})$ to achieve their covalent binding.

Finally, the functionalized nanoparticles were purified to remove the remaining reactants using a dialysis bag with a MWCO $=10,000-15,000$ for $3 \mathrm{~h}$ in $2 \mathrm{~L}$ of water followed by three more hours in $250 \mathrm{~mL}$ of Hepes buffer $20 \mathrm{mM}$, required for the in vitro cell culture manipulation. Nuclease-free water was also prepared by using $0.1 \%$ of diethylpyrocarbonate (DEPC) to ensure the removal of RNAse contamination, autoclaved and filtered before using.

To confirm the efficiency of this reaction, three tests were performed. First, the Kaiser or ninhydrin test was used, as described elsewhere, to qualitatively determine the absence of free amines in the samples (Kaiser et al., 1970). In brief, this test consists on the addition of 34 droplets (around $20 \mu \mathrm{L}$ ) of the Kaiser's solutions (a. Ninhydrin in ethanol, b. phenol in ethanol and c. $\mathrm{KCN}$ in pyridine) to about $1 \mathrm{~mL}$ of sample. After around $10 \mathrm{~min}$ of incubation of

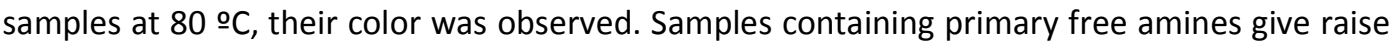
to a purple-bluish color, while samples without primary free amines resulted in a yellowwhite coloration. Approximately $1 \mathrm{~mL}$ of the sample was tested. Second, thermogravimetric assays were performed to see the changes in the mass decomposition pattern as a function of temperature, which is a feature of each material (Atkinson and Vyazovkin, 2011), using a TGA/SDTA 851e model, measuring from 20 to $550 \stackrel{\circ}{\circ}$, increasing the temperature $10 \stackrel{\circ}{\circ} / \mathrm{min}$. Third, FTIR spectra were recorded to detect modification of the chemical groups due to the covalent carbodiimide reaction, using an FTIR spectrometer Nicolet model 510, measuring from 390 to $3900 \mathrm{~cm}^{-1}$.

\subsubsection{Oligonucleotide electrostatic binding}

The electrostatic attachment of oligonucleotides to cationic nanoparticle surface was performed by mixing the cationic functionalized nanoparticles dispersed in Hepes buffer $([\mathrm{PLGA}]=2.4 \mathrm{mg} / \mathrm{mL})$ with antisense oligonucleotides, followed by their ultrasonication (Ultrasounds-H Bath model, P-Selecta, power $=200 \mathrm{~W}$ ) for $5 \mathrm{~min}$ at room temperature (25 $\mathrm{OC}$ ) and their incubation during $30 \mathrm{~min}$ at $37 \stackrel{\circ}{\circ} \mathrm{C}$. To test the formation of the complexes, hydrodynamic sizes and surfaces charges were measured as previously reported (Zamecnik and Stephenson, 1987; Fire et al., 1998). The N/P charge ratio (NP/ASO) was varied to find out the complexation ratio. To achieve the oligonucleotide protection, complexes were coated with polyethylene glycol (PEG). The coating was performed incubating the as-prepared complexes $([P L G A]=2.4 \mathrm{mg} / \mathrm{mL})$ with the PEG $(M W=400 \mathrm{Da})$ at a molar PLGA/PEG ratio of $1 / 1$ at $37 \stackrel{\circ}{ } \mathrm{C}$ for $30 \mathrm{~min}$ (Huang et al., 2008). The formation of the complexes was assessed by determining their hydrodynamic size and surface charge. 
Table 1 Characterization of the functionalized nanoparticles (surface charge and hydrodynamic radii) as a function of the molar nanoparticle/dendron ratio.

\begin{tabular}{|c|c|c|c|}
\cline { 2 - 4 } \multicolumn{1}{c|}{} & $\begin{array}{c}\text { NP/dendron } \\
\text { ratio }\end{array}$ & $\begin{array}{c}\text { Surface charge } \\
(\mathbf{m V})\end{array}$ & $\begin{array}{c}\text { Hydrodynamic } \\
\text { radii (nm) }\end{array}$ \\
\hline Without dendron & $1 / 0$ & $-18.90 \pm 1.19$ & $20.24 \pm 6.98$ \\
\hline \multirow{3}{*}{$\begin{array}{c}\text { 2nd generation } \\
\text { dendron }\end{array}$} & $1 / 1$ & $-68.05 \pm 5.03$ & $33.29 \pm 15.67$ \\
\cline { 2 - 4 } & $1 / 2$ & $-44.95 \pm 2.20$ & $25.11 \pm 0.08$ \\
\cline { 2 - 4 } & $1 / 10$ & $-9.81 \pm 0.40$ & $23.87 \pm 10.81$ \\
\cline { 2 - 4 } & $1 / 20$ & $36.00 \pm 3.00$ & $28.97 \pm 4.35$ \\
\hline \multirow{2}{*}{$\begin{array}{c}\text { 3nd generation } \\
\text { dendron }\end{array}$} & $1 / 1$ & $-11.57 \pm 0.81$ & $49.30 \pm 16.28$ \\
\cline { 2 - 4 } & $1 / 5$ & $30.78 \pm 6.71$ & $22.25 \pm 7.95$ \\
\hline
\end{tabular}

Fig. 6. Surface charge of the complexes as a function of the charge N/P (cationic functionalized NPs/oligonucleotide) ratios.

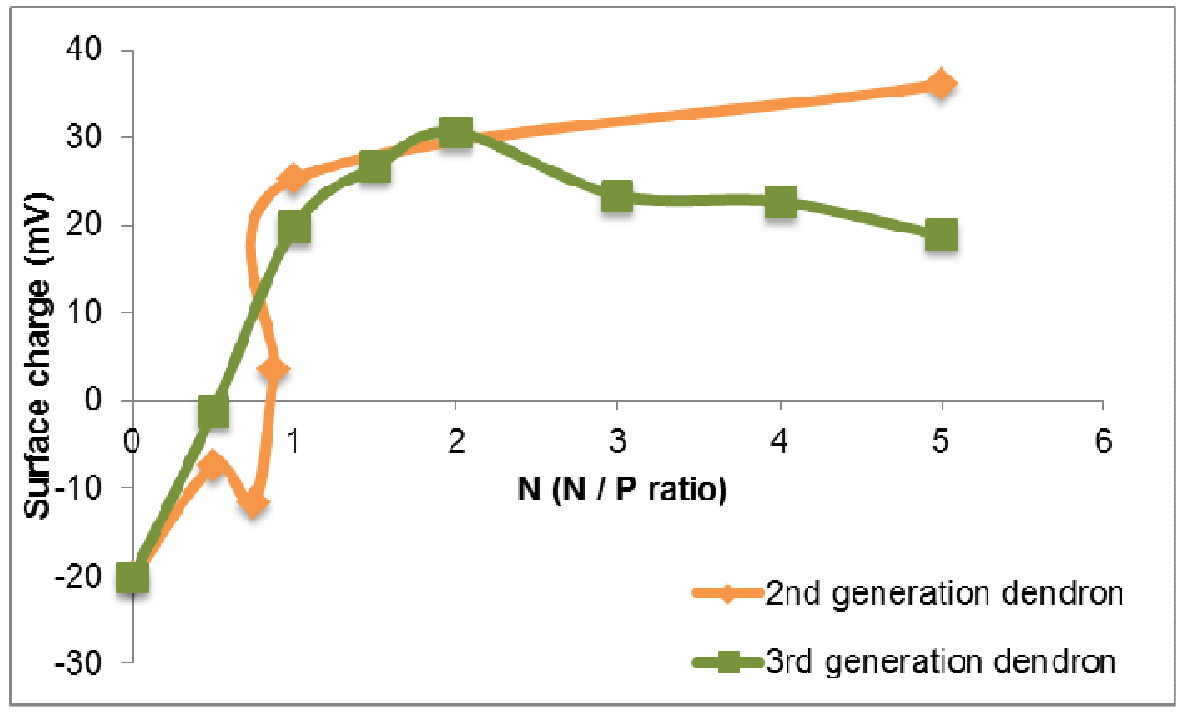

\subsubsection{Electrophoretic mobility shift assay (EMSA)}

Phosphorothioate antisense oligonucleotides $(0.5 \mu \mathrm{g})$ were mixed with increasing concentrations of cationic functionalized nanoparticles, from charge ratios of 0 to 5 . Complexes were analyzed using the electrophoretic mobility shift assay which consists on an electrophoresis on a $20 \mathrm{wt} \%$ polyacrylamide gel at $150 \mathrm{~V}$ for $8 \mathrm{~h}$ in TBE buffer. Pictures were taken using Fujifilm las- 1000 Intelligent Dark Box II using IR LAS-1000 Lite v1.2 (Grijalvo and Eritja, 2012). 


\subsubsection{In vitro cytotoxicity assay}

Two cell lines, HeLa and U87, were used for the in vitro experiments. Cell cultures were grown as described in the Supplementary information (2S).

The metabolic activity of the cells was measured after $18 \mathrm{~h}$ using the MTT test (3-(4,5dimethylthiazol-2-yl)-2,5-diphenyltetrazo- lium bromide); to determine the cytotoxic effect of nanoparticles (Putnam et al., 2001). $25 \mu \mathrm{L}$ of MTT at a concentration of $5 \mathrm{mg} / \mathrm{mL}$ in PBS were added directly in each well and cells were incubated 2 additional hours. Then, $100 \mu \mathrm{L}$ of DMSO were added. After $15 \mathrm{~min}$ incubation, absorbance was measured at $560 \mathrm{~nm}$ wavelength on microplate ELISA reader (SpectraMax M5 luminometer, Molecular devices). Results were expressed as a relative percentage to control cells (Putnam et al., 2001; Hansen et al., 1989).

The lactate dehydrogenase test (LDH) was also used to confirm the cytotoxicity results. It measures the LDH released on the medium due to the rupture of the cell membrane in case of cell death. Briefly, after following the same incubation protocol than MTT assays, $50 \mu \mathrm{L}$ of the surpernatant were placed in another plate. $150 \mu \mathrm{L}$ of PBS were added, followed by the addition of $25 \mu \mathrm{L}$ of piruvate $31 \mathrm{mM}$ and $25 \mu \mathrm{L}$ of NADH $1.3 \mathrm{mM}$. Absorbance was measured at $340 \mathrm{~nm}$ and results were expressed as a relative percentage to control cells (Putnam et al., 2001; Hansen et al., 1989; Weber et al., 2008)

\subsubsection{In vitro gene silencing}

HeLa cells were cultured as indicated (Supplementary information, 2S). Twenty-four hours before transfection at 40 to $80 \%$ confluence, cells were trypsinized and diluted 1:5 with fresh medium without antibiotics (about $1-3 \times 10^{5}$ cells $/ \mathrm{mL}$ ) and transferred to a 24-well plate (500 $\mu \mathrm{L}$ per well). After this time, $10 \mu \mathrm{L}$ of Firefly luciferase plasmid pGL3 at $100 \mu \mathrm{mol} / \mu \mathrm{L}$ and $10 \mu \mathrm{L}$ of Renilla luciferase plasmid pRL-TK at $10 \mu \mathrm{mol} / \mu \mathrm{L}$, both diluted in OptiMEM, were transfected using Lipofectamine2000 (Invitrogen) (Grijalvo and Eritja, 2012). These plasmids were incubated at 37 ㅇ $\mathrm{C}$ for $5 \mathrm{~h}$. After this time, the medium was discharged and each well was cleaned twice with PBS. $500 \mu \mathrm{L}$ of DMEM were added afterwards along with $100 \mu \mathrm{L}$ of the asprepared sample, in each well. The incubation took place for about $24 \mathrm{~h}$ at $37 \stackrel{\circ}{\circ}$. After this time, transfection cell lysates were prepared and analyzed using the Dual-Luciferase Reporter Assay System according to manufacturer's protocol. Various samples at different concentrations were studied. In addition, a positive control formulated with Lipofectamine $2000^{\circledR}$, and a negative control, formulated with scrambled ASO was added. Results are expressed as the normalized ratios between the reporter pGL3 gene and the control pRL-TK luciferase gene.

\subsubsection{In vitro haemolysis assay}

Human red blood cells (RBC) were kindly provided from blood from healthy donors. Erythrocytes were isolated by centrifugation (10 $\mathrm{min}$ at $867 \mathrm{~g}$ ) and washed trice with isotonic phosphate buffered saline (PBS; pH 7.4). The erythrocytes were then resuspended in PBS at a cell density of $8 \times 10^{9}$ cells $/ \mathrm{mL}$. $1 \mathrm{~mL}$ of each sample, at the maximum concentration of use, was placed in an eppendorf, and mixed with $100 \mu \mathrm{L}$ of RBC. The mixture was incubated at 25 o $C$, under continuous agitation for the required time (10 $\min$ or $24 \mathrm{~h})$ and then centrifuged for 
$5 \mathrm{~min}$, at $867 \mathrm{~g}$ at room temperature. The percentage of haemolysis was spectroscopically assessed by comparing the absorbance $(\lambda=540 \mathrm{~nm})$ of the samples with the negative (PBS solution) and the positive (distilled water) controls. The results are expressed as the percentage of haemolysis caused.

Table 2. Characterization of the functionalized nanoparticles with and without oligonucleotides, at N/P charge ratios of $0.75 / 1$ and $1 / 1$, for the second and third generation dendrons respectively.

\begin{tabular}{|c|c|c|c|c|}
\hline & \multicolumn{2}{|c|}{ 2nd generation dendron } & \multicolumn{2}{|c|}{ 3rd generation dendron } \\
\hline & $\begin{array}{c}\text { Dendron } \\
\text { functionalized } \\
\text { NPs }\end{array}$ & $\begin{array}{l}\text { ASO-dendron } \\
\text { multifunctiona- } \\
\text { lized NPs }\end{array}$ & $\begin{array}{c}\text { Dendron } \\
\text { functionalized } \\
\text { NPs }\end{array}$ & $\begin{array}{c}\text { ASO-dendron } \\
\text { multifunctiona- } \\
\text { lized NPs }\end{array}$ \\
\hline Hydrodynamic radius $(\mathrm{nm})$ & $28.97 \pm 4.35$ & $84.28 \pm 3.89$ & $22.25 \pm 7.95$ & $51.68 \pm 0.70$ \\
\hline Surface charge $(\mathrm{mV})$ & $36.00 \pm 3.00$ & $25.25 \pm 0.81$ & $30.78 \pm 6.71$ & $19.80 \pm 0.44$ \\
\hline Visual stability & $>3$ months & $>3$ months & $>3$ months & $>3$ months \\
\hline
\end{tabular}

\section{Results and discussion}

\subsection{Nano-emulsion formation and characterization}

Prior to nanoparticle preparation, study of nanoemulsion formation and their characterization was undertaken in the aqueous solution/polysorbate 80/[4 wt\% PLGA in ethyl acetate] system, at $25 \stackrel{\circ}{ } \mathrm{C}$. The aqueous component was an electrolyte solution (phosphate buffered saline: PBS) simulating physiological conditions. The PIC method was used to prepare nano-emulsions, as described in Section 2.2.1. Fig. 2 shows nano-emulsion formation region in the corresponding pseudoternary phase diagram. This region extends in a broad range of oil/surfactant (O/S) ratios (between 10/90 and 90/10) and aqueous contents above $70 \mathrm{wt} \%$. Nano-emulsion appearance is translucent bluish shine near the borders of the formation region and it turns transparent in the central part and as the aqueous content increases.

Nano-emulsions with $90 \mathrm{wt} \%$ of aqueous solution were selected for further studies, since they showed higher transparency than nano-emulsions with lower water contents, which is usually indicative of smaller droplet sizes and also because of their good stability assessed qualitatively. The values of hydrodynamic radii (Fig. 3), obtained by dynamic light scattering (DLS), are around $150 \mathrm{~nm}$ at low O/S ratios (below 40/60), decreasing to values below $50 \mathrm{~nm}$ at higher $O / S$ ratios and increasing to values around $90 \mathrm{~nm}$ at the highest $O / S$ ratio. The droplet size depends on the $\mathrm{O} / \mathrm{S}$ ratio of the nano-emulsion, showing higher sizes at the borders of the nano-emulsion domain due to the formation of less stable nano-emulsions. These size changes were predicted by the visual appearance of the nano-emulsions.

When using conventional (non-volatile) oil components, nano-emulsion droplet size increases with the increase in the O/S ratio (Solans and Solè, 2012; Izquierdo et al., 2005). In 
the current study, the main oil component used is a volatile solvent, ethyl acetate, partially soluble in water. As the ethyl acetate content increases at increasing $\mathrm{O} / \mathrm{S}$ ratios, more ethyl acetate diffuses to the aqueous phase, thus resulting in nano-emulsions with smaller droplet size. However, the droplet size keeps constant in a wide range of $\mathrm{O} / \mathrm{S}$ ratios. In a previous study, it was found that the droplet size of nano-emulsions, formulated using ethyl acetate and PLGA as the oil component and water as the aqueous component, tended to increase with the increase in the O/S ratio (although the increase was not pronounced) (Patil and Panyam, 2009). In contrast, in the current study, nano-emulsions are prepared using an electrolyte solution as aqueous phase, which increase the osmotic pressure difference between the aqueous and the oil phase. Therefore, diffusion of ethyl acetate through the aqueous phase is facilitated, thus the droplet size should be reduced as the $\mathrm{O} / \mathrm{S}$ ratio increases. Probably other factors (e.g., the effect of polymer as more lipophilic oil, thus preventing diffusion) counteract the expected tendency.

The polydispersity index of the nano-emulsions is lower at the central part of the nanoemulsion region (O/S ratios between $40 / 60$ and 80/20) (see Supplementary information Table $3 \mathrm{~S})$. It is worth-mentioning that the nano-emulsions reported here have smaller droplet sizes than those obtained with similar systems (Patil and Panyam, 2009; Bouchemal et al., 2004).

The surface charge of nano-emulsions is negative (Fig. 3 ) at all $\mathrm{O} / \mathrm{S}$ ratios due to the carboxylic groups of the polymer, which are deprotonated in the buffer used (neutral $\mathrm{pH}$ ) (Thomas et al., 2009). The values of the charge seem to be correlated with the sizes: the higher the hydrodynamic radius, the more charged the nano-emulsion. However, in previous reports, the electrophoretic mobility has been correlated with the adsorbed charges onto droplets surface rather than with the size (Ohshima et al., 1983). This could be due to the fact that the bigger the droplet, the higher the number of charges adsorbed into its surface; thus the zeta potential of bigger droplets is expected to be higher.

Nano-emulsions with 90 wt\% of water content, and an O/S ratio of 70/30 were used for further studies, as a compromise between small droplet size $(<40 \mathrm{~nm})$ and low surfactant content. In addition, the absolute value of their surface charge (approx. $-25 \mathrm{mV}$ ) is high enough to provide electrostatic stability (Hidalgo-Álvarez et al., 1996). The colloidal stability was assessed by macroscopic inspection of samples as well as measuring their hydrodynamic radius with time. It was observed that sedimentation occurred after five days of preparation. The sedimentation could be attributed to the partial solubility of ethyl acetate in the aqueous phase, enabling the early formation of nanoparticles from the droplets of the nano-emulsion. As nanoparticles are denser than the continuous phase, they tend to sediment. DLS measurements were in good agreement with visual observation. The size of the nano-emulsion decreases slightly with time due to the sedimentation of the early formed nanoparticles (Supplementary information, Fig. 4S).

\subsection{Nanoparticle formation and characterization}

Nanoparticles were obtained from nano-emulsions with 90 wt\% of aqueous content and 70/30 O/S ratio, by solvent evaporation (Desgouilles et al., 2003). The nanoparticle size 
was determined using two different methodologies, TEM and DLS. TEM images showed that nanoparticles have a spherical shape with smooth surfaces and sizes typically around $30 \mathrm{~nm}$, as shown in Fig. 4. DLS measurements gave hydrodynamic diameters of around $40 \mathrm{~nm}$, with polydispersity indexes around 0.2 . The differences in size obtained with both techniques were expected, as TEM measures only the hard sphere diameter while DLS measures the hydration layers of the nanoparticle. In addition, the differences could be also due to the fact that TEM image analyses are number weighted while data obtained from DLS are intensity weighted and therefore the latter supply higher size values than those by number. Comparing the hydrodynamic diameters of nano-emulsion drop-lets and nanoparticles, as expected, shrinkage due to evaporation of the solvent was observed. It is worth noting that, to our best knowledge, only two studies have reported smaller PLGA nano-particles (Pustulka et al., 2013; Panda et al., 2013) than those of this work.

Nanoparticle dispersions, as their template nano-emulsions, show a negative surface charge, of around $-20 \mathrm{mV}$, due to the negative charges of the polymer.

Concerning nanoparticle dispersion stability, no macroscopic changes were observed in two months, neither variation of the hydrodynamic radii (Supplementary information, 5S). This stability values are high if compared with other nanoparticles made of PLGA with similar size and surface charge (Pustulka et al., 2013).

The as-prepared nanoparticle dispersions were used in the subsequent studies. It should be noted that removal of the surfactant, Polysorbate 80 , used for the preparation of the template nano-emulsions is not required. It is well known that this surfactant enhances physiological membranes permeability, thus facilitating the entrance of nanoparticles through some tissues (e.g., blood-brain barrier) (Ramge et al., 2000).

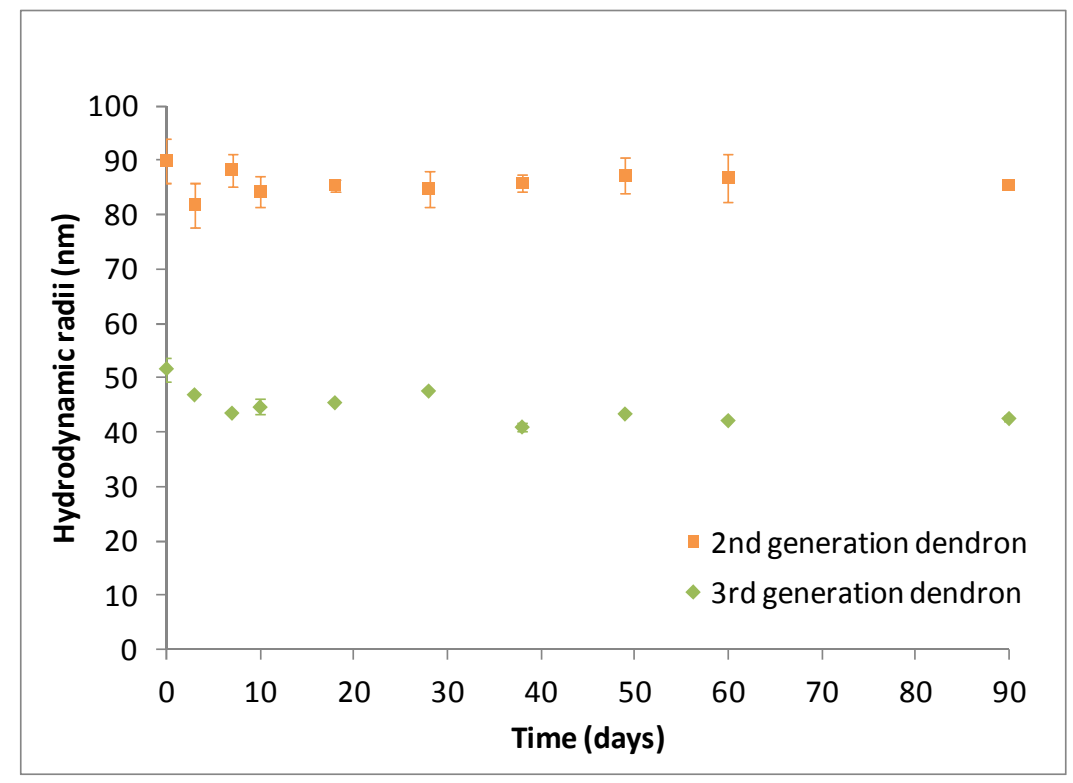

Fig. 7. Hydrodynamic radii of the complex formed using the nanoparticles functionalized with the second and third generation dendron at a N/P charge ratio of $0.25 / 1$ and $1 / 1$ respectively, as a function of time. 

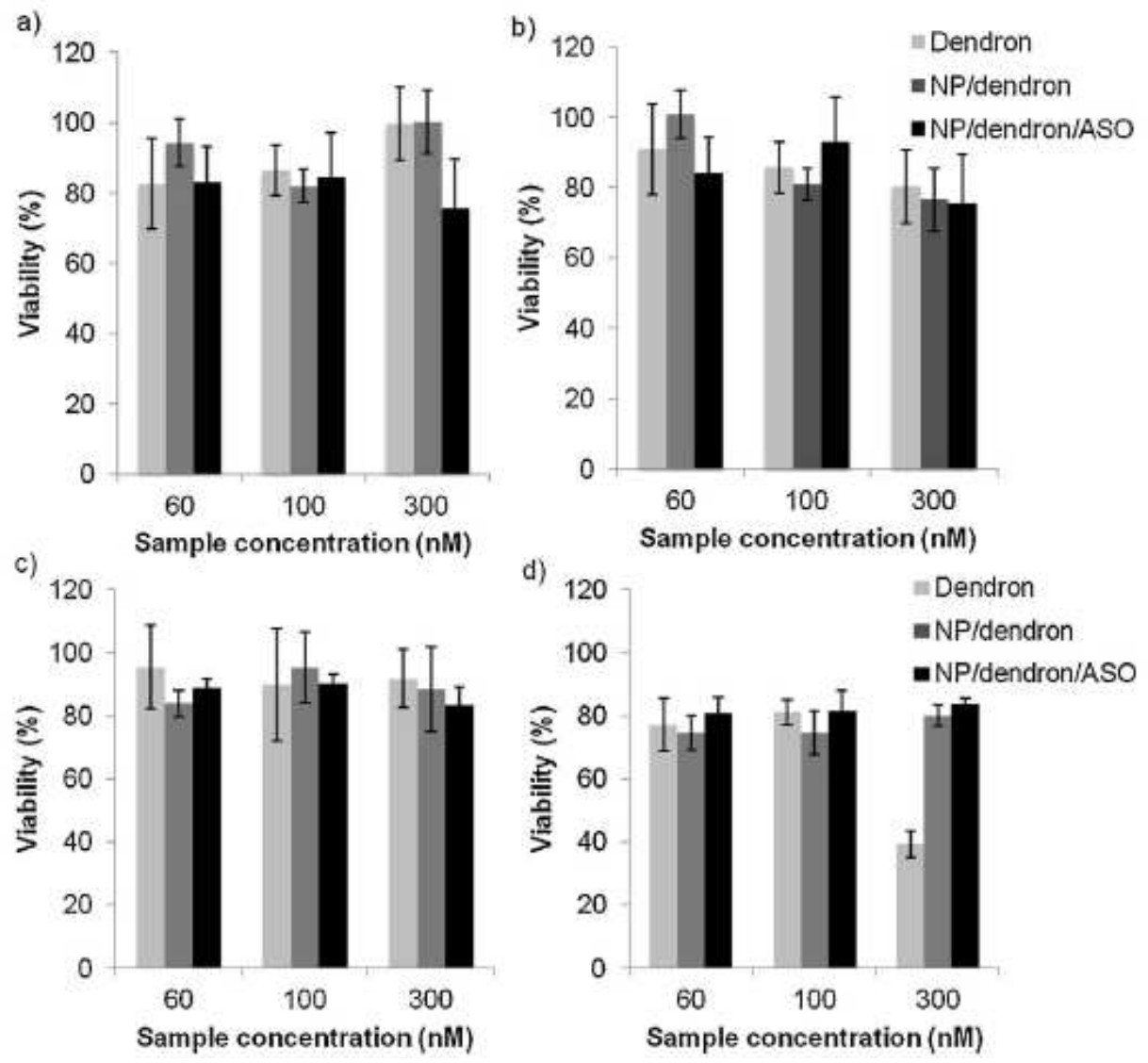

Fig. 8. Viability of HeLa cells after the incubation with the different components of the multifunctionalized nanoparticles. (a) and (c) show the results for the second generation dendron while (b) and (d) show those with the third generation dendron. (a) and (b) correspond to the MTT results while (c) and (d) correspond to the LDH results. NP/dendron ratio was $1 / 20$ for 2 nd generation dendron and $1 / 5$ for 3rd generation dendron while dendronized NP/ASO ratio was $0.75 / 1$ for 2 nd generation dendron and $1 / 1$ for 3 rd generation dendron.

\subsection{Cationic dendron functionalization of the nanoparticles}

As oligonucleotides are anionic molecules, nanoparticle surface must be positively charged for their electrostatic bind. For this purpose, cationic dendrons were covalently bound to nanoparticle surface, by using the carbodiimide reaction (Costantino et al., 2006; Betancourt et al., 2009). Two different carbosilane dendrons were tested at decreasing nanoparticle/dendron ratios: the second and the third generation constructions. To confirm nanoparticle functionalization, tests were carried out with four samples: (a) only nanoparticles; (b) only dendrons; (c) nanoparticles physically mixed with dendrons (to ensure that any reaction is not produced in absence of reactants); and (d) nanoparticles chemically bond to dendrons. In samples (c) and (d), the nanoparticle/dendron molar ratio used was $1 / 1$. The ninhydrin test, used to qualitatively asses the cationic nanoparticle-functionalization, showed a bluish-purplish coloration only of dendrons and the physical mixture; therefore, functionalized 
nanoparticles did not contained free amines, thus, it could be hypothesized that they were functionalized (Supplementary information, Fig. S6).

Thermogravimetric assays were performed to assess the covalent dendron linkage to nanoparticles, as the mass degrada-tion profile is a feature characteristic of a given compound (Bardajee et al., 2012). The first derivative of the mass degradation of all samples with the exception of the dendron, presents a main peak around $350 \stackrel{\circ}{\circ}$, which represents the degradation of the PLGA (Amna et al., 2012) (Fig. 5). The mass degradation profile of the functionalized nanoparticles consists on a main peak around 325 o $\mathrm{C}$ together with another peak around $230 \stackrel{\circ}{\circ}$, while the physical mixture consist in three overlaid peaks, around 250, 300 and 350 ㅇ. Therefore, the profile of functionalized nano-particles is different than that of the physical mixture. In the physical mixture, the degradation profile is formed by the combination of the peaks corresponding to the dendron alone and to the nanoparticles; but in the profile of the functionalized nanoparticles, the peak corresponding to nanoparticles is main-tained while the peak corresponding to the dendron fraction was shifted to 230 우 ; thus confirming the nanoparticle functionalization.

In addition, FTIR spectrograms confirmed the dendron nano-particle functionalization, since a shift on the spectra bands took place (Supplementary information, Fig. 7S).

$\mathrm{NP} /$ dendron molar ratio was varied to achieve complete functionalization of nanoparticle surface, thus obtaining cationic nanoparticles. As shown in Table 1, nanoparticle charge becomes cationic at a NP/dendron ratio of $1 / 20$ and $1 / 5$ for the second and third generation dendrons, respectively. The surface charge values for the second generation dendron grafted particles with NP/ dendron ratios of $1 / 1$ and $1 / 2$ are striking as they are lower than the particles without dendron. This could be explained considering that incorporation of the dendrons produces a slight increase in the hydrodynamic size of the complexes, which could be due to an increase of the nanoparticle itself or to an increase of the solvatation layer. If the amount of dendron is lower than that required for the cationization, the ions of the electrolyte (PBS) solution used for nanoparticle preparation could remain around the nanoparticles, with a different compactation, increasing the amount of ions inside the solvatation layer. In this case, an increase in the absolute value of the surface charge would be expected. The complexation ratio is higher for the second generation dendron which means that a higher amount of this dendron is needed to achieve cationic complexes. This result was already expected, since each molecule of this dendron has less positive charges, consequently, more amount of the dendron is required to obtain cationic nanoparticles.

At the appropriate N/D ratios, the hydrodynamic radii of both complexes did not experienced a significant variation (Supple-mentary Fig. 8S). Both complexes were stable for, at least, three months, as assessed by visual observation of the dispersions and droplet size determination as a function of time. Both dendronized nanoparticles at the corresponding $\mathrm{NP} /$ dendron molar ratio were chosen to perform the in vitro studies.

Most of the existing bibliography on this topic is based in dendron functionalization of inorganic nanoparticles (Cho et al., 2011) and only few studies report the use of polymeric nanoparticles (Sakthivel and Florence, 2003). To our knowledge, the only study on the use of PLGA nanoparticles for dendron functionalization (Costantino et al., 2006) obtained 
$\mathrm{NP} /$ dendron ratios higher than those ones reported here. Low ratios are advantageous, because the increase of the nanoparticle size is nearly zero (Table 1 ), thus maintaining the nanoparticle size in the range of intravenously administrable nanosystems. Moreover, the positive charges of the carbosilane dendrons used in this work do not depend on $\mathrm{pH}$, since they are ammonia groups without protonation groups, while other dendrons such those based on PAMAM suffer from autoprotonation which could negatively influence the definition of N/D ratios (Pistolis et al., 1999).

\subsection{Nanoparticle - antisense oligonucleotide (ASO) complexation}

Nanoparticles with cationic surface charge are able to form complexes by electrostatic interaction with antisense oligonucleo- tides. The complexes were tested using both dendrons, varying the functionalized nanoparticles/antisense oligonucleotide (N/P) charge ratio in order to obtain complexes with as less charge as possible since they allow for longer blood circulation times and reduced plasma protein adsorption (Sakthivel and Florence, 2003). Fig. 6 shows the surface charge of the formulated complexes as a function of the N/P ratio, testing second and third generation dendrons. For both complexes, the N/P complexation charge ratios (null surface charge) are below $1 / 1$, indicating that the amount of required nanoparticle as a function of oligonucleotide concentration is low.

To further define the exact complexation charge ratio, EMSA experiments were carried out (Supplementary information, Fig. 8S). The results confirmed the N/P complexation ratios found by $Z$ potential measurements. Characterization (size and surface charge) of the ASOdendron functionalized nanoparticle, just after preparation, is summarized in Table 2, which for comparative purposes also shows the characterization of dendron functionalized nanoparticles. The size of the nanoparticles increases after the complexation with oligonucleotides, according to previous bibliography (Huang et al., 2008) (Table 2 and Table 9S), which was attributed to a swelling of nanoparticles due to their more hydrophilic surface. This increase is double for the third generation dendron but quadruple for the second generation dendron; which could be due to the more amount of oligonucleotide required to compensate the positive charges in the second generation dendron-functionalized nanoparticles. Concerning the surface charge, after the complexation with oligonucleotides (for both dendrons), the surface charge is reduced, due to the compensation of the positive charges of the dendrons by the oligonucleotides. Size and charge variation may represent a confirmation of the complex formation. Table 2 also shows the stability of the nanoparticles, assessed by visual observation.

The stability was also assessed by measuring hydrodynamic radii of the complexes with time. The as-prepared complexes are stable for, at least, three months (Fig. 7).

\subsection{Cytotoxicity assay}

The in vitro cytotoxicity of the dendrons, the cationic functionalized nanoparticles and ASO-complexes was tested as a function of the concentration of ASO-complexes using the MTT colorimetric assay (Putnam et al., 2001; Hansen et al., 1989) as well as the LDH assay (Weber et al., 2008). The concentrations of all samples are referred to the sample concentration required for a desired oligonucleotide concentration. The results of viability of HeLa after their 
incubation with the samples (Fig. 8) showed that for all the components studied, except for the third generation dendron, the relative viabilities are higher than $75 \%$, and the cytotoxicity does not vary increasing the sample concentration up to a concentration of oligonucleotide of $300 \mathrm{nM}$ (Fig. 8). In addition, assays performed on U87 cells were also used to assess the cytotoxicity of the formulated complexes (Supplementary information, Fig. 10S). Both results confirmed that the as-prepared complexes are non-cytotoxic, thus, they can be used for biomedical applications.

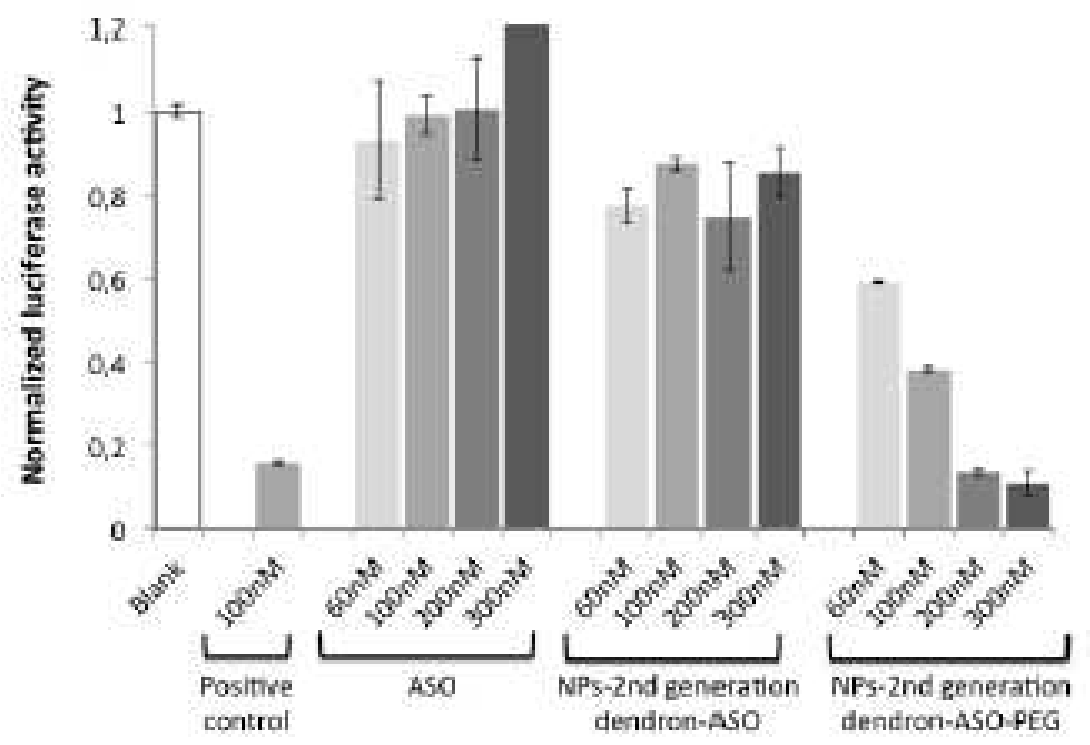

Fig. 9. Percentage of luciferase activity inhibition for the complex formulated with the second generation dendron at N/P ratio of $0.75 / 1$ and increasing concentrations. Lipofectamine 2000 was used as the positive control and an scrambled ASO as the negative control.

\subsection{Gene silencing efficiency in cell culture}

The formulated complexes could be used as non-viral gene delivery vectors using the intravenous route of administration. The gene silencing efficiency must be measured to ensure an intracellular oligonucleotide concentration sufficient to produce an in vivo activity. Blank samples and antisense oligonucleotides alone (wild type (WT) samples), without forming complexes with nanoparticles were used as controls. First, the gene silencing efficiency was tested using both complexes in serum-free conditions, The N/P ratios tested were those ones around the complexation. The inhibition is decreased up to $38 \%$ only using the complex formulated with the second generation dendron at an oligonucleotide concentration of $60 \mathrm{nM}$ and a N/P ratio of $0.75 / 1$, indicating that the gene silencing efficiency is $62 \%$ (Supplementary information, Fig. 11S). This optimal formulation was chosen for further studies, while the complexes formulated using the third generation dendrons were discarded. Their lower silencing efficiencies were attributed to the slight toxicity found for the third generation dendron in the LDH test (Fig. 8D). 
With the selected complex, the gene silencing efficiency was also studied with $10 \%$ of fetal bovine serum (FBS), to simulate physiological conditions. To avoid the nanoparticle blood clearance by the immune system, polyethylene glycol (PEG) coating has been described to enhance steric stability of nanoparticles against opsonization and phagocytosis. Thus, nanoparticle circulation time in the bloodstream will be longer, enabling them to arrive to their targets (Mao et al., 2001). The PEGylation of nanoparticles (with the second generation dendron and with ASO) resulted in hydrodynamic radii of around $85 \mathrm{~nm}$ and negative surface charges $(-10 \mathrm{mV})$, in agreement with previous studies (Huang et al., 2008). As displayed in Fig. 9 , the gene silencing efficiency in the presence of FBS is reduced, as expected. Different oligonucleotide concentrations were tested. The best result was obtained at 60 and $200 \mathrm{nM}$, with a gene silencing efficiency of nearby $25 \%$. The gene silencing efficiency is really increased when nanoparticles were coated with PEG, achieving promising gene silencing efficiencies, with values up to $90 \%$ of gene silencing, comparable with those of commercial vectors (positive control of Fig. 9). This could be due to the presence of the PEG, which may protect the antisense oligonucleotide from nucleases degradation.

The good gene silencing efficiencies are usually correlated with small sizes. However, in the presence of the serum, sizes are expected to increase due to the serum adsorption onto complex surfaces (Huang et al., 2013). Serum proteins have negative charges, like antisense oligonucleotides. For this reason, they can compete with the oligonucleotides for the electrostatic binding to the cationic nanoparticle surface. The increase of the complexes size combined with the competition between the oligonucleotides and the plasma proteins for the nanoparticle surface could be the cause of the reduced gene silencing efficiencies.

This theory is in good agreement with the results obtained using the PEG coating. Since the oligonucleotides electrostatically bind to nanoparticle surface are protected with poly(ethylene glycol), the plasma proteins cannot interact with nanoparticle surface, thus enabling increased gene silencing efficiencies, really high if compared with the previous bibliography (Torchilin, 2006).

To the best of our knowledge, the use of PLGA nanoparticles prepared by a low-energy nano-emulsification approach, containing the hybrid dendron-oligonucleotide has never been reported yet in gene silencing studies. These results could open a new window in the search of new non-viral carriers, since they have similar transfection efficiencies than those of commercial vectors, but they are non-cytotoxic (Fig. 8) neither hemolytic (results shown below) at the required concentrations of use.

\subsection{In vitro haemolysis tests}

Since the formulated complexes were designed to be used as non-viral gene delivery systems for the administration of genetic material using the intravenous route of administration; the blood toxicity should be taken into account. For this reason, the haemolytic activity of the formulated complexes using the second generation dendron was studied. The results (Table 3 ) showed that none of the formulated complexes produced 
haemolysis after their incubation with red blood cells during $10 \min$ or $24 \mathrm{~h}$, at the concentration of use.

Since these complexes are formulated using biocompatible and biodegradable materials and they do not show haemolytic activity for at least $24 \mathrm{~h}$, they are not expected to be toxic once intravenously injected.

\begin{tabular}{|c|c|c|c|c|}
\hline Sample & Incubaton tme & Haemohreir & Vinual appearance & Miarogaphy \\
\hline $\begin{array}{l}\text { NP } \\
\text { NP-dendron } \\
\text { NP-dendran-NSO } \\
\text { NP-dendron+ } N S O-P E C\end{array}$ & $10 \mathrm{~min}$ & or & & \\
\hline $\begin{array}{l}\text { NP } \\
\text { NP-dendron } \\
\text { NP-dendron- }-150 \\
\text { NP-dendron+ } A 5 O+P E C\end{array}$ & $24 \mathrm{~h}$ & $\infty$ & & \\
\hline Negasve contral (PRS solintion) & $10 \mathrm{~min} / 24 \mathrm{~h}$ & $\sigma r$ & & \\
\hline Poutive control (diratled wza) & $10 \min / 24 \mathrm{~h}$ & or & & \\
\hline
\end{tabular}

Table 3 Results of the haemolytic tests for different incubation times and different samples. All samples were studied at the maximum concentration of use ([ASO] $=300 \mathrm{nM}$; $[\mathrm{PLGA}]=1.275$ $\mathrm{mM}$; [dendron] $=25.5 \mathrm{mM}$ ). For each group, an example of the visual appearance of the samples after the final centrifugation and a micrograph of the erythrocytes once incubated with the samples is given. The two first examples correspond to the nanoparticles + dendron + ASO sample. NP/dendron ratio was $1 / 20$ for 2 nd generation dendron and 1/5 for 3rd generation dendron while dendronized NP / ASO ratio was $0.75 / 1$ for 2 nd generation dendron and $1 / 1$ for 3 rd generation dendron.

\section{Conclusions}

In this work, polymeric nanoparticles have been prepared by nano-emulsion templating. A low-energy emulsification method, the phase inversion composition (PIC) method was used for nano-emulsion formation. An electrolyte solution simulating the physiological conditions was the aqueous phase, the emulsifier was a nonionic ethoxylated surfactant, widely used in clinical tests, and the oil phase consisted on PLGA polymerdissolved in ethyl acetate, a GRAS solvent belonging to the class 3 of the ICH guidelines. Nano-emulsions formed in a wide range of $\mathrm{O} / \mathrm{S}$ ratios at high water contents. Template nano-emulsions with 90 wt\% of aqueous phase content, and an $\mathrm{O} / \mathrm{S}$ ratio of 70/30 were selected as a compromise between small sizes $(<40 \mathrm{~nm})$ and low surfactant contents. Nanoparticles were prepared by solvent evaporation of nano-emulsions, showing diameters of around $30 \mathrm{~nm}$, negative surface charges due to the carboxylic groups of the polymer and stabilities of around three months. 
The as-prepared nanoparticles were dendron-functionalized in order to obtain cationic surfaces. Cationic carbosilane dendrons of the second and the third generation, previously defined as non-toxic were used. A ratio of $1 / 20$ was required for the whole cationization using the second generation dendron, while using $1 / 5$ ratio was enough for the third generation dendron. The complexation of dendron functionalized nanoparticles with antisense oligonucleotides by electrostatic interactions evidenced that low complexation ratios for both dendrons (specially indicated concerning toxicity issues) were required. The complexation ratio using the second generation dendron is lower (0.25/1) than using the third generation dendron (1/1). Characterization of these complexes showed sizes higher than the dendronized nanoparticles, thus indicating the formation of the complexes. Toxicity experiments showed that the complexes were non-cytotoxic as well as non-haemolytic. Gene silencing assays were performed with both complexes. The best results were obtained using the second generation dendronized nanoparticles, achieving gene silencing efficiencies as high as $90 \%$ when the gene material was protected with PEG. These results offer new perspectives in the use of nanoparticles containing cationic dendrons and oligonucleotides as novel promising non-viral gene delivery systems.

\section{Acknowledgements}

Financial support from MINECO (grants CTQ2011-29336-CO3- O1, CTQ2010-20541 and CTQ2011-23245); Generalitat de Catalunya (grant 2009-SGR-961, 2009-SGR-208), Consortium NANO- DENDMED ref S2011/BMD-2351 (CAM) and CIBER-BBN are acknowledged. CIBER-BBN is an initiative funded by the VI National R\&D\&I Plan 2008-2011, Iniciativa Ingenio 2010, Consolider Program, CIBER Actions and financed by the Instituto de Salud Carlos III with assistance from the European Regional Development Fund. Cristina Fornaguera is grateful to AGAUR for their Predoctoral Fellowship (grant FI-DGR 2012). Authors acknowledge Adele Alagia for her kind support in the preparation of cell culture experiments. Authors also acknowledge Prof. Pilar Vinardell and Dr. Montserrat Mitjans for their kind support in the haemolysis experiments. Authors also acknowledge Dr. J. Blanco for the kind gift of the U87 cells.

\section{Appendix A.}

Supplementary data Supplementary data associated with this article can be found, in the online version, at http://dx.doi.org/10.1016/j. ijpharm.2014.11.031.

\section{References}

Amna, T., Hassan, M.S., Nam, K.T., Nim, Y.Y., Barakat, N.A.M., Khil, N.S., Kim, H.Y., 2012. Preparation, characterization and cytotoxicity of CPT/Fe2O3-embedded PLGA ultrafinecomposite fibers: a synergistic approach to develop promising anticancer materials. Int. J. Nanomed. 7, 1659-1670.

Atkinson, J.L., Vyazovkin, S., 2011. Non-oxidative thermal degradation of poly (glycidol), poly(glycidol)-g-lactide, and poly(glycicol)-g-glycolide. Macromol. Chem. Phys. 212, 2103-2113. 
Bardajee, G.R., Hooshyar, Z., Rezanezhad, H., 2012. A novel and green biomaterial based silver nanocomposite hydrogel synthesis characterization and antibacterial effect. J. Inorg. Biochem. 117, 367-373.

Basarkar, A., Singh, J., 2007. Nanoparticulate systems for polynucleotide delivery. Int. J. Nanomed. 2 (3), 353-360.

Betancourt, T., Byrne, J.D., Sumaryo, N., Crowder, S.W., Kadapakkam, M., Patel, S., Casciato, S., Brannon-Peppas, L., 2009. PEGilation strategies for active targeting of PLA/PLGA nanoparticles. J. Biomed. Mat. Res. A 91A (1), 263-273.

Bouchemal, K., Briançon, S., Perrier, E., Fessi, H., 2004. Nano-emulsion formulation using spontaneous emulsification: solvent, oil and surfactant optimisation. Int. J. Pharm. 280, 241251.

Calderó, G., Solans, C., 2012. Use of nano-emulsions as templates for the preparation of polymeric nanoparticles. In: Perosa, A., Selva, M. (Eds.), Green Processes. Volume 8: Green Nanoscience. Wiley-VCH Verlag, Weinheim, Germany, pp. 165-174.

Calderó, G., García-Celma, M.J., Solans, C., 2011. Formation of polymeric nano-emulsions by low-energy method and their use for nanoparticle preparation. J. Colloid. Interface Sci. 353, 406-411.

Chavany, C., Le Doan, T., Couvreur, P., Puisieux, F., Hélène, C., 1992. Polyalkylcyanoacrylate nanoparticles as polymeric carriers for antisense oligonucleotides. Pharm. Res. 9, 441-449.

Cho, T.J., Zangmeister, R.A., MacCuspie, R.I., Patri, A.K., Hackley, V.A., 2011. Newkome-type dendron-stabilized gold nanoparticles synthesis reactivity, and stability. Chem. Mat. 23, 26652676.

Costantino, L., Gandolfi, F., Bossy-Nobs, L., Tovi, G., Gurny, R., Rivasi, F., Vandelli, M.A., Forni, R., 2006. Nanoparticulate drug carriers based on hybrid poly(lactide-co-glycolide)-dendron structures. Biomaterials. 27, 4635-4645.

Desgouilles, S., Vauthier, C., Bazile, D., Vacus, J., Grossiord, J.L., Veillard, M., Couvreur, P., 2003. The design of nanoparticles obtained by solvent evaporation: a comprehensive study. Langmuir 19 (22), 9504-9510.

Dinarvand, R., Sepehri, N., Manoochehri, S., Rouhani, H., Atyabi, F., 2011. Polylactide- coglycolide nanoparticles for controlled delivery of anticancer agents. Int. J. Nanomed. 6, 878895.

Fattal, E., Vauthier, C., Ayne, I., Nakada, Y., Lambert, G., Malvy, C., Couvreur, P., 1998. Biodegradable polyalkylcyanoacrylate nanoparticles for the delivery of oligonucleotides. J. Control Release 53, 137-143.

Fire, A., Xu, S., Montgomery, M.K., Kostas, S.A., Driver, S.E., Mello, C.C., 1998. Potent and specific genetic interference by double-stranded RNA in Caenorhabditis elegans. Nature 391, 806-811. 
Fuentes-Paniagua, E., Peña-González, C.E., Galán, M., Gómez, R., de la Mata, F.J., SánchezNieves, J., 2013. Thiol-ene synthesis of cationic carbosilane dendrons: a new family of synthons. Organometallics 32, 1789-1796.

Giacca, M., Zacchigna, S., 2012. Virus-mediated gene delivery for human gene therapy. J. Control Release 161, 377-388.

Grijalvo, S., Eritja, R., 2012. Synthesis and in vitro inhibition properties of oligonucleotide conjugates carrying amphipathic proline-rich peptide derivatives of the sweet arrow peptide (SAP). Mol. Divers. 16, 307-317.

Hansen, M.B., Nielsen, S.G., Berk, K., 1989. Re-examination and further development of a precise and rapid dye method for measuring cell growth/cell kill. J. Immunol. Methods 119 (2), 203-210.

Hidalgo-Álvarez, R., Martín, A., Fernandez, A., Bastos, D., Martínez, F., de las Nieves, F. J., 1996. Electrokinetic properties, coloidal stability and aggregation kinetics of polymer colloids. Adv. Colloid Interface Sci. 67, 1-118.

Huang, Y., Chen, J., Chen, X., Gao, J., Liang, W., 2008. PEGylated synthetic surfactant vesicles (Niosomes): novel carriers for oligonucleotides. J. Mater. Sci. Mater. Med. 19 (2), 607-614.

Huang, S.J., Wang, T.P., Lue, S.I., Wang, L.F., 2013. Pentablock copolymers of Pluronic F127 and modified poly(2-dimethyl amino)ethyl methacrylate for internalization mechanism and gene transfection studies. Int. J. Nanomed. 8, 2011-2027.

Izquierdo, P., Feng, J., Esquena, J., Tadros, T., Dederen, J.C., García-Celma, M.J., Azemar, N., Solans, C., 2005. The influence of surfactant mixing ratio on nano- emulsion formation by the PIT method. J. Colloid Interface Sci. 285 (1), 388- 394.

Kaiser, E., Colescott, R.L., Bossinger, C.D., Cook, P.I., 1970. Color tests for detection of free terminal amine groups in the solid-phase synthesis of peptides. Anal. Biochem. 34, 595-598.

Kumar, M.N.V.R., Bakowsky, U., Lehr, C.M., 2004. Preparation and characterization of cationic PLGA nanospheres as DNA carriers. Biomaterials. 25 (10), 1771-1777.

Landfester, K., 2001. The generation of nanoparticles in miniemulsions. Adv. Mater. 13 (10), 765-768.

Li, C., Guo, T., Zhou, D., Hu, Y., Zhou, H., Wang, S., Chen, J., Zhang, Z., 2011. A novel glutathione modified chitosan conjugate for efficient gene delivery. J. Control Release 154, 177-188.

Loke, S.L., Stein, C.A., Zhang, X.H., Mori, K., Nakanishi, M., Subasinghe, C., Cohen, J.S., Necker, L.M., 1989. Characterization of oligonucleotide transport into living cells. PNAS 86 (10), 34743478.

Mao, H.Q., Roy, K., Troung-Le, V.L., Janes, K.A., Lin, K.Y., Wang, Y., August, J.T., Leong, K.W., 2001. Chitosan-DNA nanoparticles as gene carriers: synthesis, characterization and transfection efficiency. J. Control Release 70 (3), 399- 421. 
Meyer, O., Kirpotin, D., Hong, K., Sternberg, B., Park, J.W., Woodlee, M.C., Papahadjopoulos, D., 1998. Cationic liposomes coated with polyethylene glycol as carriers for oligonucleotides. J. Biol. Chem. 273, 15621-15627.

Newland, B., Zheng, Y., Jin, Y., Abu-Rub, M., Cao, H., Wang, W., Pandit, A., 2012. Single cyclized molecule versus single branched molecule: a simple and efficient $3 \mathrm{~d}$ knot polymer structure for nonviral gene delivery. JACS 134, 4782-4789.

Ohshima, H., Healy, T.W., White, L.R., 1983. Faraday transactions 2: molecular and chemical physics. J. Chem. Soc. 79, 1613-1628.

Ortega, P., Bermejo, J.F., Chonco, L., de Jesus, E., de la Mata, F.J., Fernández, G., Flores, J. C., Gómez, R., Serramía, M.J., Muñoz-Fernandez, M.A., 2006. Novel water- soluble carbosilane dendrimers: synthesis and biocompatibility. Eur. J. Inorg. Chem. 7, 1388-1396.

Panda, J.J., Varshney, A., Chauhan, V.S., 2013. Self-assembled nanoparticles based on modified cationic dipeptides and DNA: novel systems for gene delivery. J. Nanobiotechnol. 11, 18.

Patil, J., Panyam, J., 2009. Polymeric nanoparticles for siRNA delivery and gene silencing. Int. J. Pharm. 367, 195-203.

Pistolis, G., Malliaris, A., Tsiourvas, D., Paleos, C.M., 1999. Poly(propyleneimine) dendrimers as pH-sensitive controlled-release systems. Eur. J. Chem. 5 (5), 1440-1444.

Puras, G., Mashal, M., Zárate, M., Agirre, M., Ojeda, E., Grijalvo, S., Eritja, R., Diaz- Tahoces, A., Martínez Navarrete, G., Avilés-Trigueros, M., Fernández, E., Pedraz, J. L., 2014. A novel cationic niosome formulation for gene delivery to the retina. J. Control Release 174, 27-36.

Pustulka, K.M., Wohl, A.R., Lee, H.S., Michel, A.R., Han, J., Hoye, T.R., McCormick, A.V., Panyam, J., Macosko, C.W., 2013. Flash nanoprecipitation: particle structure and stability. Mol. Pharm. 10, 4367-4377.

Putnam, D., Gentry, C.A., Pack, D.W., Langer, R., 2001. Polymer-based gene delivery with low cytotoxicity by a unique balance of side-chain termini. PNAS. 98 (3), 1200-1205.

Ramge, P., Unger, R.E., Oltrogge, J.B., Zenker, D., Begley, D., Kreuter, J., von Briesen, H., 2000. Polysorbate-80 coating enhances uptake of polybutylcyanoacrylate (PBCA)-nanoparticles by human and bovine primary brain capillary endothelial cells. Eur. J. Neuro Sci. 12 (6), 19311940.

Rowe, R.C., Sheskey, P.J., Owen, S.C., 2006. Handbook of Pharmaceutical Excipients, fifth ed. Pharmaceutical-Press.

Sadurní, N., Solans, C., Azemar, N., García-Celma, M.J., 2005. Studies on the formation of O/W nano-emulsions, by low-energy emulsification methods, suitable for pharmaceutical applications. Eur. J. Pharm. Sci. 26 (5), 438-445.

Sakthivel, T., Florence, A.T., 2003. Adsorption of amphipathic dendrons on polystyrene nanoparticles. Int. J. Pharm. 254, 23-26. Solans, C., Solè, I., 2012. Nano-emulsions: production by low-energy methods. Curr. Opin. Colloid Interface Sci. 17 (5), 246-254. 
Solans, C., Izquierdo, P., Nolla, J., Azemar, N., Garcia-Celma, M.J., 2005. Nano- emulsions. Currt. Opin. Colloid Interface Sci. 10, 102-110.

Tahara, K., Sakai, T., Yamamoto, H., Takeuchi, H., Hirashim, N., Kawashim, H., 2009. Improved cellular uptake of chitosan-modified PLGA nanospheres by A549 cells. Int. J. Pharm. 382, 198204.

Thomas, C., Gupta, V., Ahsan, F., 2009. Influence of surface charge of PLGA particles of recombinant hepatitis $B$ surface antigen in enhancing systemic and mucosal immune responses. Int. J. Pharm. 379 (1), 41-50.

Torchilin,V.P.,2006.Multifunctionalnanocarriers.Adv.DrugDeliv.Rev.58,1532-1555.

Vauthier, C., Bouchemal, K., 2009. Preparation and manufacture of polymeric nanoparticles. Pharm. Res. 26 (5), 1025-1058.

Weber, N., Ortega, P., Clemente, M.I., Shcharbin, D., Bryszewska, M., de la Mata, F.J., Gómez, R., Muñoz-Fernández, M.A., 2008. Characterization of carbosilane dendrimers as effective carriers of siRNA to HIV-infected lymphocytes. J. Control Release 123 (1), 55-64.

Zamecnik, P.C., Stephenson, M.L., 1987. Inhibition of Rous sarcoma virus replication and cell transformation by a specific oligodeoxynucleotide. PNAS 75, 280-284. 
Novel non-viral gene delivery systems composed of carbosilane dendron functionalized nanoparticles prepared from nano-emulsions as non-viral carriers for antisense oligonucleotides

Cristina Fornaguera, Santiago Grijalvo, Marta Galán, Elena Fuentes-Paniagua, Francisco Javier de la Mata, Rafael Gómez, Ramon Eritja, Gabriela Calderó, Conxita Solans

\section{SUPPLEMENTARY INFORMATION}

\section{Explanation 1S:}

Preparation of the nanoparticles for TEM measurements

Prior to characterization, nanoparticle dispersions were concentrated by ultracentrifugation $\left(27,000 \mathrm{~g}, 2^{\circ} \mathrm{C}, 1\right.$ hour $)$. The supernatant was removed and nanoparticle pellets were washed once with water and centrifuged again after vigorous stirring. This procedure was repeated four times. One drop of the concentrated nanoparticle dispersion was placed on a copper grid. After 1 minute, the excess of the sample was blotted with filter paper and the grid containing the sample was stained negatively with phosphotungstic acid (PTA) solution (2wt.\% in MilliQ water) for another minute. Excess solution was then removed to obtain a proper negative staining. Finally, grids were placed in a Petri dish and let dry at room temperature. 


\section{Explanation 2S:}

Cell growth

HeLa cell cultures were grown in 96 -well plates at an initial density of 6,000 cells per well in $200 \mu \mathrm{L}$ of growth medium for 24 hours. The growth medium consists on Dulbecco's modified Eagle's medium (DMEM) supplemented with 10 wt. \% FBS (fetal bovine serum). After 4 hours of incubation at $37^{\circ} \mathrm{C}$, the medium containing the incubated samples was removed and replaced for growth medium.

U87 cell cultures wer grown as explained for HeLa cells, with the exception of the growth medium, that consisted on Dulbecco's modified Eagle's medium with Nutrient Mixture F12 (DMEM/F12) supplemented with 10 wt. \% FBS (fetal bovine serum). 
Table 3S: Polydispersity indexes of nano-emulsions prepared with $90 \mathrm{wt} \%$ of water content, as a function of the O/S ratio.

\begin{tabular}{cc}
\hline O/S ratio & PDI \\
\hline $20 / 80$ & 0.44 \\
\hline $30 / 70$ & 0.40 \\
\hline $40 / 60$ & 0.45 \\
\hline $50 / 50$ & 0.35 \\
\hline $60 / 40$ & 0.27 \\
\hline $70 / 30$ & 0.27 \\
\hline $80 / 20$ & 0.27 \\
\hline $85 / 15$ & 0.25 \\
\hline $87,5 / 12,5$ & 0.45 \\
\hline
\end{tabular}




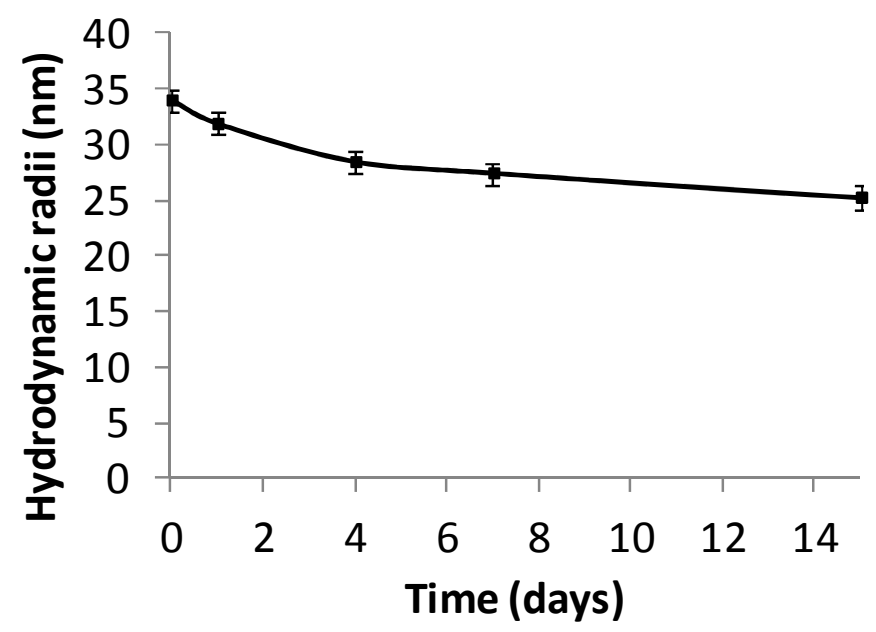

Figure 4S: Hydrodynamic radii of the nano-emulsion with $90 \mathrm{wt} \%$ of water content and $70 / 30 \mathrm{O} / \mathrm{S}$ ratio, as a function of time. 


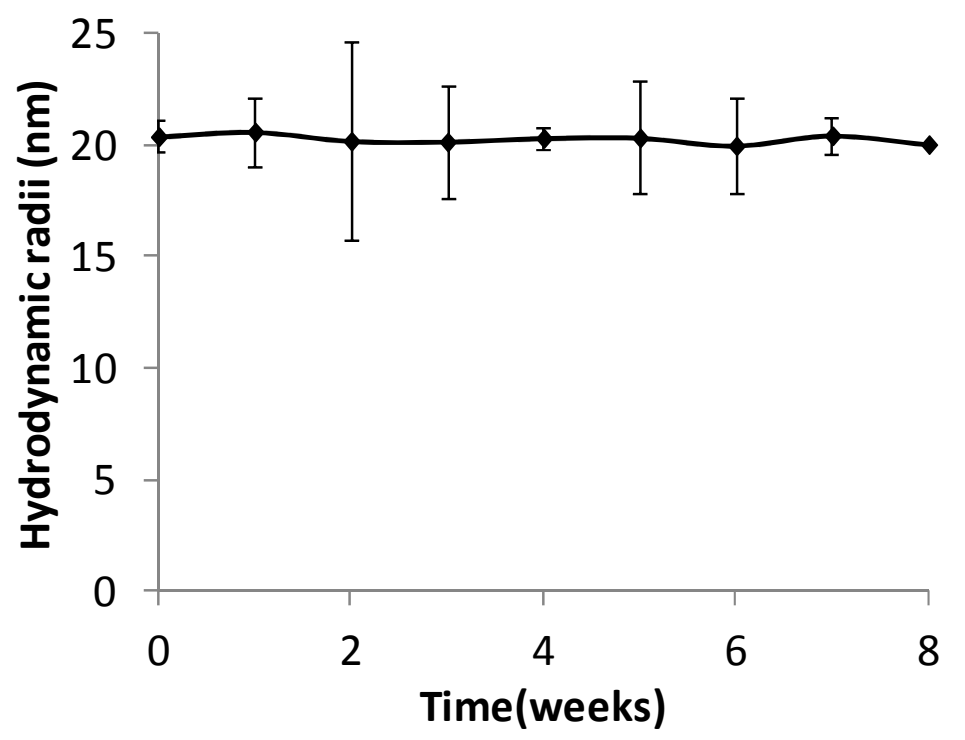

Figure 5S: Hydrodynamic radii of the nanoparticles prepared from the nano-emulsion with $90 \mathrm{wt} \%$ of water content and $70 / 30 \mathrm{O} / \mathrm{S}$ ratio, as a function of time. 


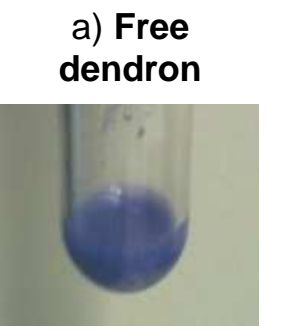

b) NPs

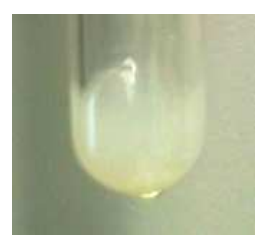

C) Dendron + NPs

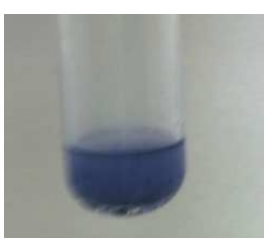

d) Functionalized NPs

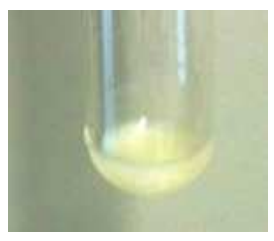

Figure 6S: Visual aspect of samples after performing the qualitative Kaiser test to detect free amines. Blue/purple coloration indicates a positive result, while white/yellow indicates a negative result.

The ninhydrin reaction, known as Kaiser test, was used to test the cationic nanoparticle-functionalization. It detects the presence of primary free amines. Since free dendrons have primary amines at the focal point, they show a positive result in the ninhydrin reaction (Figure 5a) as well as their physical mixture with nanoparticles (Figure 5c). As nanoparticles do not have free amines, the result of the test is negative (Figure 5b). After the carbodiimide reaction and purification of the functionalized nanoparticles, the Kaiser test was also negative, indicating that effectively, the nanoparticles were functionalized and no free dendrons remained (Figure 5d). 
a)

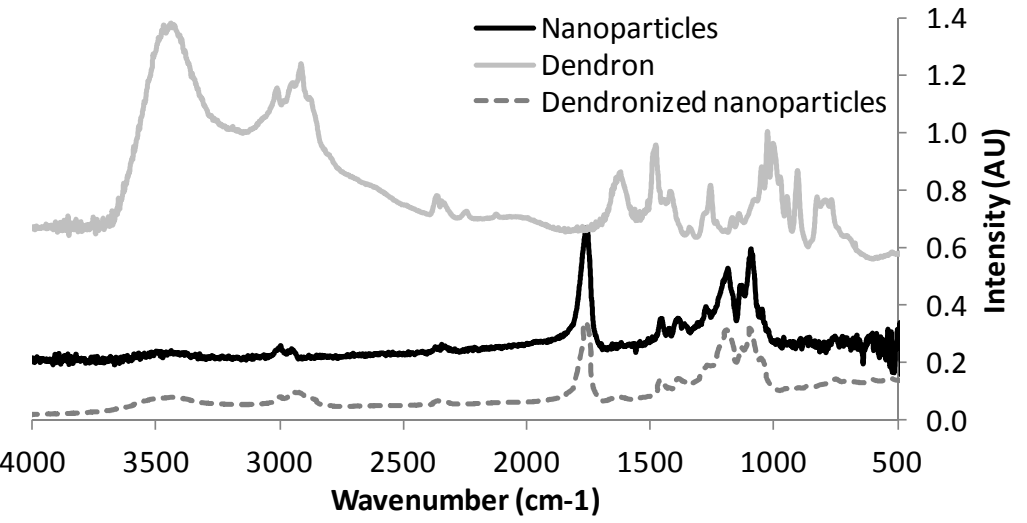

b)

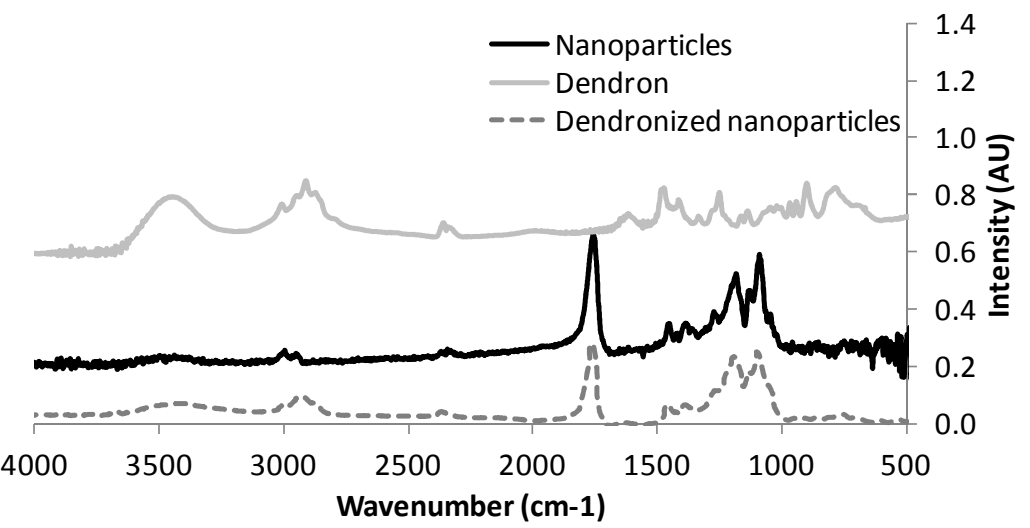

Figure 7S: FTIR spectra from nanoparticles, dendrons and dendronized nanoparticles with a) second generation dendron and b) third generation dendron.

FTIR characterization revealed a shift of the peaks tending to the apparition of the amide bands $\left(-\mathrm{NH}\right.$ around $\left.3500-1600 \mathrm{~cm}^{-1}\right)$ in the dendronized nanoparticles, where the bands corresponding to the dendron (carbosilane groups around $1050 \mathrm{~cm}^{-1}$ ) are not observed due to the superposition with the bands from the PLGA polymer. The covalent attachment can be confirmed (refusing a mixture between both components) due to the absence of the bands corresponding to the dendron (e.g. primary amine at 1623 $\mathrm{cm}^{-1} \quad$ and $-\mathrm{CH}_{3}$ at 1475 $\left.\mathrm{cm}^{-1}\right)$. 
a)

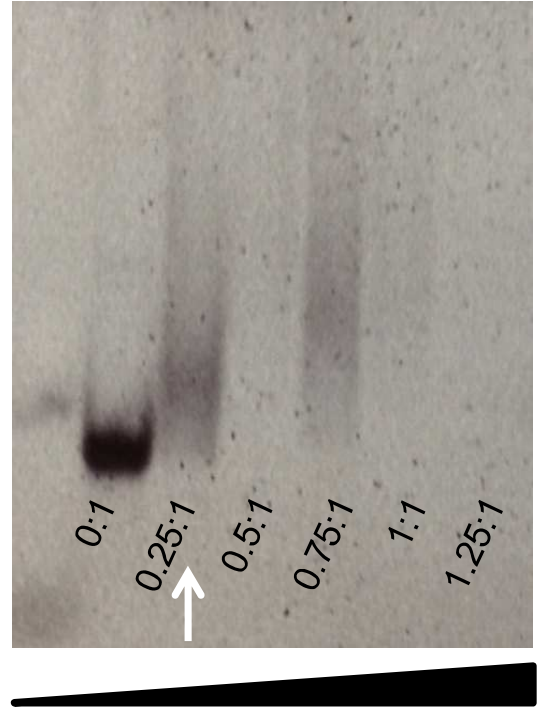

$\mathrm{N}$ : P ratio b)

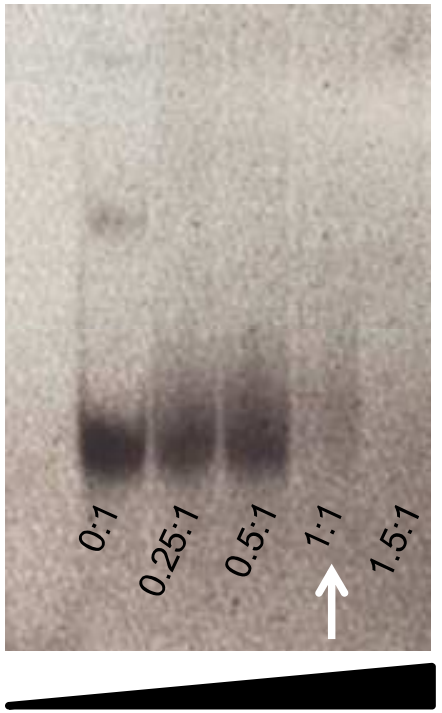

$\mathrm{N}: \mathrm{P}$ ratio

Figure 8S: EMSA results of a) 2nd generation dendron and b) 3rd generation dendron oligonucleotide functionalized nanoparticles as a function of the N/P ratio. Arrows indicate the N/P charge complexation ratio.

EMSA experiments were carried out around the complexation ratios found with the $Z$ potential measurements. Well defined spots appear when the sample charge is positive, turning to diffused retarded marks as the charge approaches to zero, in which case the complexation takes place. at N/P ratios of $0.25 / 1$ and $1 / 1$ for the nanoparticles functionalized with the second and third generation dendrons, respectively, complexation starts. These results indicate that a higher concentration of oligonucleotide is required using the second generation dendron for the same nanoparticle concentration. This result was already expected, as the surface charge of nanoparticles functionalized with the second generation dendron is higher (Table 1). Indeed, the amount of the second generation dendron is expected to be fourfold higher than that for the third generation dendron for the nanoparticle functionalization, and the third generation dendron has only twice the charge of the second generation one. 
Table 9S: Summary of the nanoparticle droplet size (by DLS) as a function of the decorating compounds.

\begin{tabular}{ccc}
\hline Sample & $\begin{array}{c}\text { Hydrodynamic } \\
\text { radius (nm) }\end{array}$ & PDI \\
\hline NPs & $20.24 \pm 6.98$ & 0.34 \\
\hline NPs-2nd generation dendron & $28.97 \pm 4.35$ & 0.36 \\
\hline NPs-3rd generation dendron & $22.25 \pm 7.95$ & 0.36 \\
\hline NPs-2nd generation dendron-ASO & $84.28 \pm 3.89$ & 0.33 \\
\hline NPs-3rd generation dendron-ASO & $51.68 \pm 0.70$ & 0.30 \\
\hline NPs-2nd generation dendron-ASO-PEG & $85.29 \pm 2.75$ & 0.37 \\
\hline
\end{tabular}




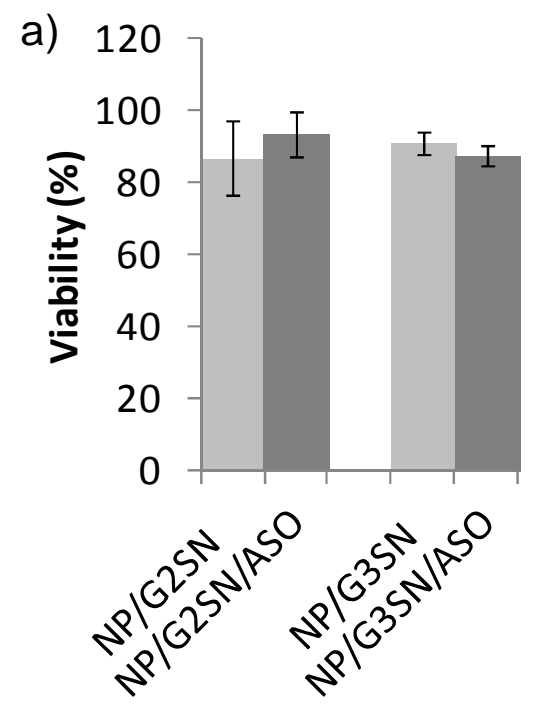

Samples
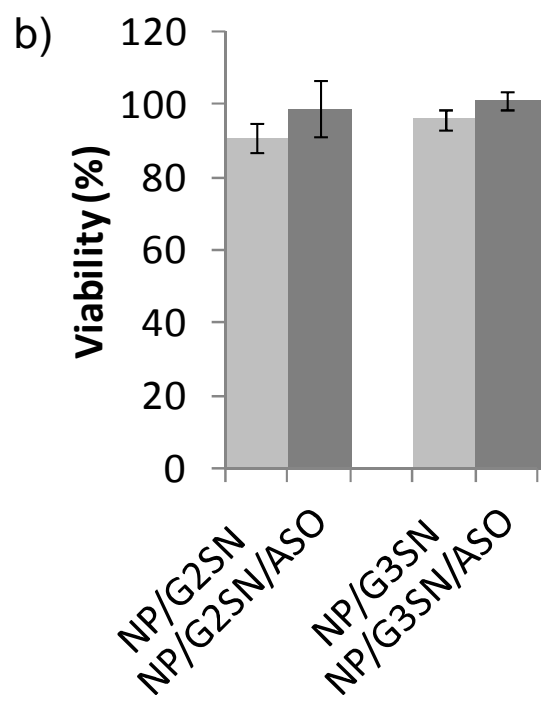

Samples

Figure 10S: Viability of U87 cells after the incubation with the different components of the multifunctionalized nanoparticles by means of the: a) MTT test and b) LDH test. 


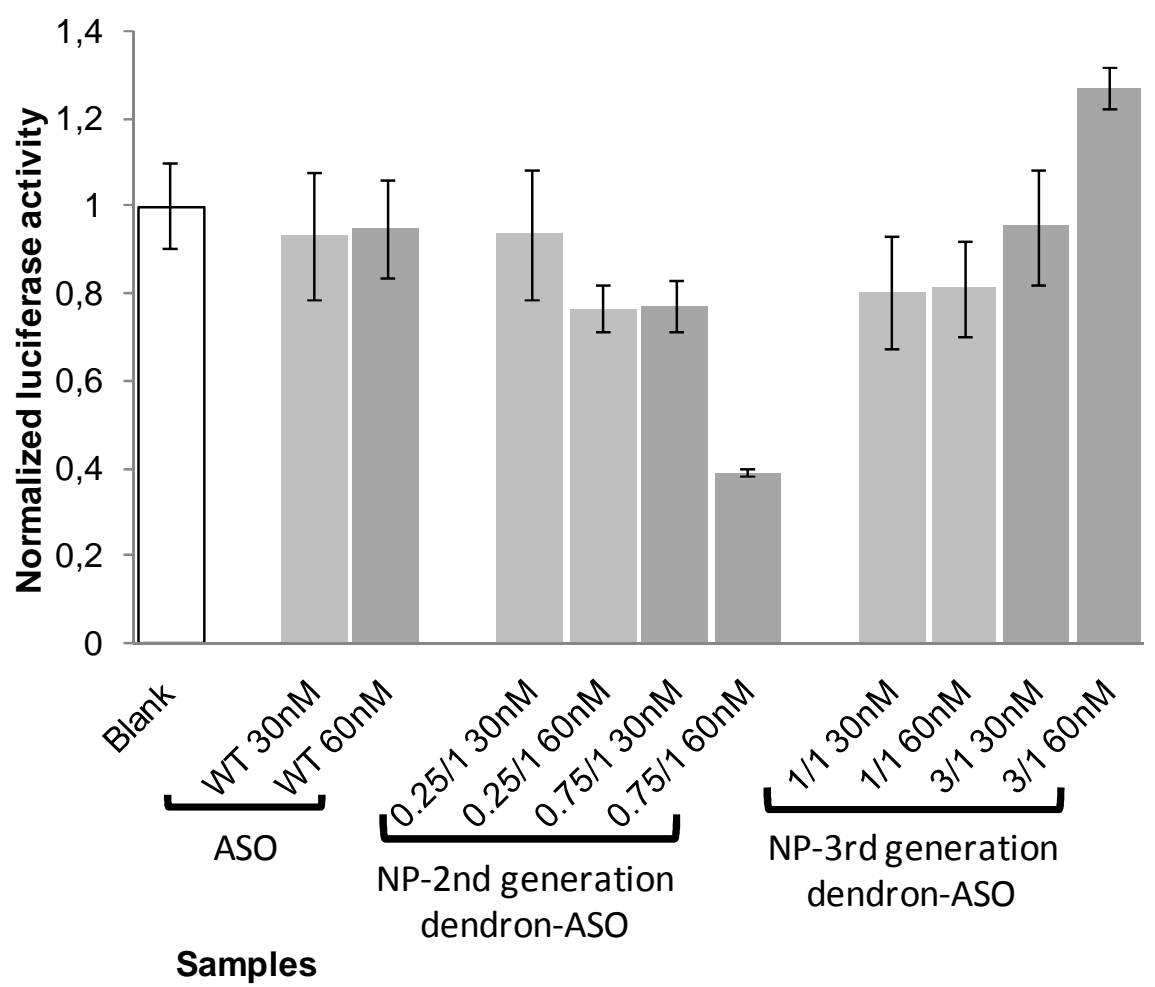

Figure 11S: Percentage of luciferase activity inhibition for the different samples assayed in serum-free conditions at different N/P (cationic functionalized nanoparticles/oligonucleotide) ratios and increasing concentrations. Results are presented as normalized percentages of the inhibition of the luciferase activity.

Gene silencing efficiency is higher for the $2^{\text {nd }}$ generation dendrojn, which could be attributed to the slight toxicity found for the $3^{\text {rd }}$ generation dendron in the LDH assay (Figure 7D).

Gene silencing efficiency depended also on the N/P ratio. The higher gene silencing efficiency was found, for each generation, at the N/P ratio of complexation found by EMSA experiments (Figure 8S). 